Article

\title{
Fuzzy Results for Finitely Supported Structures
}

\author{
Andrei Alexandru ${ }^{1}$ and Gabriel Ciobanu ${ }^{2, *(D)}$ \\ 1 Institute of Computer Science, Romanian Academy, 700505 Iaşi, Romania; \\ andrei.alexandru@iit.academiaromana-is.ro \\ 2 Faculty of Computer Science, Alexandru Ioan Cuza University, 700506 Iaşi, Romania; \\ * Correspondence: gabriel@info.uaic.ro
}

check for updates

Citation: Alexandru, A.; Ciobanu, G. Fuzzy Results for Finitely Supported Structures. Mathematics 2021, 9, 1651. https://doi.org/10.3390/math9141651

Academic Editor: Michael Voskoglou

Received: 31 May 2021

Accepted: 7 July 2021

Published: 13 July 2021

Publisher's Note: MDPI stays neutral with regard to jurisdictional claims in published maps and institutional affiliations.

\begin{abstract}
We present a survey of some results published recently by the authors regarding the fuzzy aspects of finitely supported structures. Considering the notion of finite support, we introduce a new degree of membership association between a crisp set and a finitely supported function modelling a degree of membership for each element in the crisp set. We define and study the notions of invariant set, invariant complete lattices, invariant monoids and invariant strong inductive sets. The finitely supported (fuzzy) subgroups of an invariant group, as well as the $L$-fuzzy sets on an invariant set (with $L$ being an invariant complete lattice) form invariant complete lattices. We present some fixed point results (particularly some extensions of the classical Tarski theorem, Bourbaki-Witt theorem or Tarski-Kantorovitch theorem) for finitely supported self-functions defined on invariant complete lattices and on invariant strong inductive sets; these results also provide new finiteness properties of infinite fuzzy sets. We show that apparently, large sets do not contain uniformly supported, infinite subsets, and so they are invariant strong inductive sets satisfying finiteness and fixed-point properties.
\end{abstract}

Keywords: invariant set; $L$-fuzzy set; $T$-fuzzy set; invariant complete lattice; invariant strong inductive set; fixed points; $S$-finite support principle; uniformly supported set

MSC: AMS 2020 Subject Classification; 03E30; 03E72; 08A72

\section{Introduction}

\subsection{General Aspects}

Lotfi Zadeh published in 1965 his pioneering article[1] that has over 100,000 citations today. The theory of fuzzy sets was introduced in Zermelo-Fraenkel set theory (ZF) as a framework for studying the concepts of vagueness and uncertainty. Each element of a fuzzy set has a certain degree of membership belonging to the real interval $[0,1]$. Fuzzy aspects can be applied in various fields of mathematics and computer science such as algebra, logic, analysis, operational research, control theory, decision theory, artificial intelligence and expert systems [2].

We extended the classical approach of fuzzy theory to characterise fuzzy sets over finitely supported structures. The finitely supported sets and structures are related to permutation models from Zermelo-Fraenkel set theory with atoms (ZFA) and to admissible sets (particularly to hereditary finite sets) described in [3]. They were originally introduced by Fraenkel, Lindenbaum and Mostowski during the period 1922-1938 in order to prove the independence of the axiom of choice and the other axioms in ZFA. The axioms of the recently introduced Fraenkel-Mostowski set theory are precisely the axioms of ZFA set theory together with an additional axiom for finite support. They are involved in the (hierarchical) construction of finitely supported sets; hereditary finitely supported sets are the sets constructed with respect to Fraenkel-Mostowski axioms over an infinite family of basic elements called atoms. 


\subsection{Motivation and Novelties}

The motivation for studying finitely supported sets comes from the idea of dealing in a discrete manner with infinite algebraic structures (hierarchically constructed from atoms) by analysing their finite supports. Even thoughwe admit the existence of infinite atomic sets for such a structure, we are focused only on a finite family of its elements (namely, its 'finite support', a set which is able to characterise the entire structure).

The finitely supported structures can be described both in the ZF framework and in the ZFA framework. We follow the approach presented in [4] as an alternative to the Fraenkel-Mostowski set theory, and work over the classical ZF set theory. We define invariant sets as ZF sets equipped with actions of the group of all permutations of a certain fixed set $A$ (formed by elements whose internal structure is ignored, called atoms) satisfying a certain finite support requirement. The related requirement states that any element of an invariant set is left unchanged under the effect of each permutation of $A$ that fixes pointwise, finitely,many atoms. Finitely supported sets are defined as finitely supported elements in the powerset of an invariant set. Finitely supported structures are finitely supported sets endowed with finitely supported internal laws (that are supported as functions, i.e., as subsets in a Cartesian product of invariant set); more details can be found in [5,6]. The theory of finitely supported sets allows the computational study of structures which may be infinite, but contain enough symmetries such that they can be concisely represented [6].

Finitely supported sets include ZF sets that are trivial invariant sets. However, translating ZF results in the framework of finitely supported sets is not an easy task because the family of finitely supported sets is not closed under subset constructions (there exist subsets of finitely supported sets that fail to be finitely supported; for instance, the ZF infinite and coinfinite subsets of the set $A$ of all atoms). In order to prove results for the finitely supported sets, we cannot use results from ZF without reformulating them with respect to the finite support requirement. As a consequence, there exist results which are valid in $\mathrm{ZF}$, but fail to be valid for finitely supported sets (e.g., choice principles and Stone duality) [6].

Our main purpose is to analyse whether a non-atomic ZF result can be adequately reformulated by replacing 'non-atomic ZF element/set/structure' with 'atomic, finitely supported element/set/structure'. A proof of a result in the framework of finitely supported structures should involve only finitely supported constructions even in the intermediate steps. The meta-theoretical techniques for the translation of a result from non-atomic structures to finitely supported atomic structures are based on a refinement of the finite support principle from [4], presented in [6] and called the ' $S$-finite support principle' claiming that 'for any finite set $S \subseteq A$, anything that is definable under the rules in higher-order logic from $S$-supported structures by involving only $S$-supported constructions is also $S$-supported'. The formal use of this principle implies a hierarchical construction of the support of a structure by employing, step-by-step, the supports of the substructures of a related structure.

Here, we present an overview of results dealing with fuzzy aspects of finitely supported structures (fss). Essentially, by employing the notion of 'finite support', we extend the fuzzy aspects from a finite framework to a (finitely supported) infinite one. Using specific proof techniques that are extensively presented in $[5,6]$, we obtain new algebraic properties of the fuzzy sets over fss, including some that cannot be obtained in ZF set theory. We introduce a new (infinite) fss degree of membership association, and connect it to the notions of invariant monoids and invariant complete lattices. We also show that the family of finitely supported (fuzzy) subgroups of an invariant group forms an invariant complete lattice, and that the family of finitely supported fuzzy normal subgroups forms an invariant modular lattice. We present some fixed point theorems for finitely supported structures that are preserving the validity the classical fixed point theorems, but also some fixed point properties of the finitely supported algebraic structures without corresponding results in ZF set theory. As applications of the fixed point theorems, we present some examples of finitely supported ordered structures for which these results can be used; in particular, properties of $L$-fuzzy and $T$-fuzzy sets defined in the framework of finitely 
supported structures, where $L$ is an invariant complete lattice and $T$ is an invariant strong inductive set.

\section{Finitely Supported Sets: Preliminaries}

We consider a fixed infinite ZF set $A$ without involving any internal structure for its elements. As usual, a transposition is a function $(x y): A \rightarrow A$ defined by $(x y)(x)=y$, $(x y)(y)=x$ and $(x y)(z)=z$ for $z \neq x, y$. The permutations of $A$ are bijections of $A$ generated by finitely composing many transpositions, i.e., bijections of $A$ leaving unchanged all but the finiteelements of $A$. The set of all permutations of $A$ is denoted by $S_{A}$. We proved in [5] that any finitely supported bijection of $A$ should be necessarily a permutation of $A$, i.e., it should be expressed as a finite composition of transpositions. Thus, the notions 'bijection of $A^{\prime}$ and 'permutation of $A^{\prime}$ coincide in finitely supported structures.

Definition 1. Let $X$ be a ZF set.

1. An $S_{A}$-action on $X$ is a group action of $S_{A}$ on $X$. An $S_{A}$-set is a pair $(X, \cdot)$, where $X$ is a $Z F$ set and $\cdot: S_{A} \times X \rightarrow X$ is an $S_{A}$-action on $X$.

2. Let $(X, \cdot)$ be an $S_{A}$-set. We say that $S \subset A$ supports $x$ (or $x$ is $S$-supported) if for each $\pi \in \operatorname{Fix}(S)$ we have $\pi \cdot x=x$, where $\operatorname{Fix}(S)=\{\pi \mid \pi(a)=$ a for all $a \in S\}$. An element which is supported by a finite subset of atoms is called finitely supported.

3. Let $(X, \cdot)$ be an $S_{A}$-set. We say that set $X$ is an invariant set whenever for each $x \in X$ there is a finite set $S_{x} \subset A$ supporting $x$.

4. Let $X$ be an $S_{A}$-set, and $x \in X$. If there is a finite set supporting $x$, then a least finite set $\operatorname{supp}(x)$ supporting $x$ [5], defined as the intersection of all sets supporting $x$, which is called the support of $x$. An empty supported element is equivariant; $z \in X$ is equivariant if and only if $\pi \cdot z=z$ for all $\pi \in S_{A}$.

Let $(X, \cdot)$ and $(Y, \diamond)$ be $S_{A}$-sets. According to [6], the set $A$ of atoms is an invariant set with the $S_{A}$-action $: S_{A} \times A \rightarrow A$ defined by $\pi \cdot a:=\pi(a)$ for all $\pi \in S_{A}$ and $a \in A$. Moreover, $\operatorname{supp}(a)=\{a\}$ for each $a \in A$. If $\pi \in S_{A}$ and $x \in X$ is finitely supported, then $\pi \cdot x$ is finitely supported and $\operatorname{supp}(\pi \cdot x)=\{\pi(u) \mid u \in \operatorname{supp}(x)\}:=\pi(\operatorname{supp}(x))$. The Cartesian product $X \times Y$ is an $S_{A}$-set with the $S_{A}$-action $\otimes$ defined by $\pi \otimes(x, y)=$ $(\pi \cdot x, \pi \diamond y)$ for all $\pi \in S_{A}$ and all $x \in X, y \in Y$. For $(X, \cdot)$ and $(Y, \diamond)$ invariant sets, $(X \times Y, \otimes)$ is also an invariant set. The powerset $\wp(X)=\{Z \mid Z \subseteq X\}$ is an $S_{A}$-set with the $S_{A}$-action $\star: S_{A} \times \wp(X) \rightarrow \wp(X)$ defined by $\pi \star Z:=\{\pi \cdot z \mid z \in Z\}$ for all $\pi \in S_{A}$ and $Z \subseteq X$. For an invariant set $(X, \cdot), \wp_{f s}(X)$ denotes the set formed from those subsets of $X$ that are finitely supported in the sense of Definition 1(2) as elements in $\wp(X)$ with respect to the action $\star ;\left(\wp_{f_{s}}(X),\left.\star\right|_{\wp_{f s}}(X)\right)$ is also an invariant set, where $\left.\star\right|_{\wp_{f s}}(X)$ represents the action $\star$ restricted to $\wp_{f_{S}}(X)$. Non-atomic sets are trivially invariant, i.e., they are equipped with the action $(\pi, x) \mapsto x$.

A subset $Z$ of an invariant set $(X, \cdot)$ is called finitely supported if and only if $Z \in \wp_{f s}(X)$, i.e., if and only if $Z$ is finitely supported as an element of the $S_{A}$-set $\wp(X)$ with respect to the action $\star$ defined on $\wp(X)$. A subset $Z$ of $X$ is uniformly supported if all of its elements are supported by the same finite set of atoms (elements of $A$ ). Certainly, a finite subset of an invariant set should be uniformly supported (by the union of the supports of its elements), but there may exist invariant sets that do not contain uniformly supported, infinite subsets, as we will prove below.

Let us notice that not any subset of an invariant set is finitely supported. For instance, if $X \subset A$ and $X$ is finite, then it is finitely supported with $\operatorname{supp}(X)=X$. If $Y \subseteq A$ and $Y$ is cofinite (i.e., its complement is finite), then it is finitely supported with $\operatorname{supp}(Y)=A \backslash Y$. Whenever $Z \subseteq A$ is neither finite nor cofinite, then $Z$ is not finitely supported. It is proven that a subset of $A$ is finitely supported if and only if it is either finite or cofinite [7]. Moreover, if $\pi$ is a permutation of $A$ and $X$ is a subset of an $S_{A}$-set $Y$, then $\pi \star X=X$ if and only if $\pi \star X \subseteq X$, considering $\star$ defined on $\wp(Y)$. As a consequence of the previous definitions, a subset $Z$ of an invariant set $(X, \cdot)$ is supported by a finite set $S \subseteq A$ if and only 
if $\pi \star Z \subseteq Z$ for all $\pi \in F i x(S)$, i.e., if and only if $\pi \cdot z \in Z$ for all $z \in Z$ and $\pi \in$ Fix (S) (this happens because permutations of atoms are of a finite order). If $X$ is an invariant set, its finite powerset $\wp_{\text {fin }}(X)$ (namely, the set of all finite subsets of $X$ ) and its cofinite powerset $\wp_{\text {cofin }}(X)$ (namely, the set of all cofinite subsets of $X$ ) are equivariant subsets of $\wp_{f s}(X)$, meaning that they are themselves invariant sets having the restrictions of the action $\star$ on $\wp_{f \mathcal{S}}(X)$. In [6], we proved that $\operatorname{supp}(X)=\cup_{x \in X} \operatorname{supp}(x)$ whenever $X$ is a uniformly supported subset of an invariant set.

As functions are specific relations (i.e., subsets of a Cartesian product of two sets), for two invariant sets $(X, \cdot)$ and $(Y, \diamond), Z$, a finitely supported subset of $X$, and $T$, a finitely supported subset of $Y$, we say that a function $f: Z \rightarrow T$ is finitely supported if $f \in$ $\wp_{f_{S}}(X \times Y)$. Note that $Y^{X}$ is an $S_{A}$-set with the $S_{A}$-action $\widetilde{\star}: S_{A} \times Y^{X} \rightarrow Y^{X}$ defined by $(\pi \widetilde{\star} f)(x)=\pi \diamond\left(f\left(\pi^{-1} \cdot x\right)\right)$ for all $\pi \in S_{A}, f \in Y^{X}$ and $x \in X$. A function $f: X \rightarrow Y$ is finitely supported (in the sense of the above definition) if and only if it is finitely supported with respect to the permutation action $\widetilde{\star}$. The set of all finitely supported functions from $Z$ to $T$ is denoted by $T_{f s}^{Z}$. As an immediate characterisation, a function $f: Z \rightarrow T$ is supported by a finite set $S \subseteq A$ if and only if for all $x \in Z$ and all $\pi \in \operatorname{Fix}(S)$ we have $\pi \cdot x \in Z$, $\pi \diamond f(x) \in T$ and $f(\pi \cdot x)=\pi \diamond f(x)$.

An invariant, partially ordered set (invariant poset) is an invariant set $(P, \cdot$, ) equipped with an equivariant partial order relation $\sqsubseteq$ on $P$. A finitely supported, partially ordered set is a finitely supported subset $Q$ of an invariant set together with a finitely supported partial order relation. An invariant complete lattice is an invariant partially ordered set $(L, \cdot, \sqsubseteq)$ such that every finitely supported subset $X \subseteq L$ has a least upper bound with respect to the order relation $\sqsubseteq$. It is proven [6] that in an invariant complete lattice, every finitely supported subset $X \subseteq L$ has a greatest upper bound with respect to the order relation $\sqsubseteq$. A finitely supported complete lattice is a finitely supported subset $L$ of an invariant set, equipped with a finitely supported order $\sqsubseteq$ such that every finitely supported subset of $L$ has a least upper bound with respect to $\sqsubseteq$.

In both [5,6], several examples of invariant/finitely supported partially ordered sets are presented. For example, if $X$ is an invariant/finitely supported set, then $\left(\wp_{f_{s}}(X), \star, \subseteq\right)$ is an invariant/finitely supported complete lattice. Here, we focus on the fuzzy theory over invariant sets, mainly presenting the results of [6-10].

\section{Fuzziness over Invariant Sets}

Let us consider a set $U$ called the universal set (or the universe of discourse). Recall that a crisp set $Z$ in the universe of discourse $U$ can be described by mentioning all of its members or by specifying the properties that have to be be satisfied by its members. The theory of fuzzy sets is a generalisation of this classical view: a fuzzy set is represented by a subset $Z$ of the universal set $U$ which has associated a related membership function generalising the characteristic function from the classical set theory. More exactly, the membership function associated to $Z$ could take any values in the interval $[0,1]$ (modelling a certain degree of membership), while the classical characteristic function of $Z$ can only take two values: 0 (for non-membership) and 1 (for membership). Fuzzy sets over infinite invariant sets were introduced and studied first in [8], and then extended in [9].

Definition 2. A fuzzy set over the invariant set $(U, \cdot)$ is a finitely supported subset $Z$ of $(U, \cdot)$ together with a finitely supported membership function $\mu_{Z}: U \rightarrow[0,1]$.

We say simply that $\left(Z, \mu_{Z}\right)$ is a fuzzy set over $(U, \cdot)$. In our approach, a fuzzy set over the invariant set $U$ is a (finitely supported) element in the invariant set $\left(\wp_{f s}(U) \times\right.$ $\left.[0,1]_{f_{S}}^{U}, \otimes\right)$. It is easy to see that in such a Cartesian pair, there is no precise fss association between the crisp finitely supported subset of $U$ and the related finitely supported function belonging to $[0,1]_{f s}^{U}$. Therefore, we allow more than one finitely supported membership function to be associated with the same finitely supported subset of $U$. 
We decided to not yet define such an fss association because it is not necessary to assume the existence of an explicit finitely supported relation on $\wp_{f_{s}}(U)$ and $[0,1]_{f_{s}}^{U}$ for proving the properties of fuzzy sets for fss. On the other hand, the case when certain fss relations are defined between $\wp_{f s}(U)$ and $[0,1]_{f s}^{U}$ is analysed later in Section 4.

In the theory of fuzzy sets in ZF, we have two useful notions: $\alpha$-cut and fuzzy support. For fss, an $\alpha$-cut of a fuzzy set $\left(Z, \mu_{Z}\right)$ over the invariant set $(U, \cdot)$ is a crisp set $Z_{\alpha}$ containing all the elements in $U$ with membership values greater than or equal to $\alpha$, i.e., $Z_{\alpha}=\left\{z \in U \mid \mu_{Z}(z) \geq \alpha\right\}$.

Proposition 1. Any $\alpha$-cut $Z_{\alpha}$ of a fuzzy set $\left(Z, \mu_{Z}\right)$ over the invariant set $(U, \cdot)$ is a finitely supported subset of $U$ with the property that $\operatorname{supp}\left(Z_{\alpha}\right) \subseteq \operatorname{supp}\left(\mu_{Z}\right)$.

Definition 3. The fuzzy support of a fuzzy set $\left(Z, \mu_{Z}\right)$ over the invariant set $(U, \cdot)$ (also called the algebraic support of $\left.\mu_{Z}\right)$ is a crisp set $F Z S\left(Z, \mu_{Z}\right)$ containing all the elements in $U$ with membership values greater than 0 , i.e., $F Z S\left(Z, \mu_{Z}\right)=\left\{z \in U \mid \mu_{Z}(z)>0\right\}$.

We prove (similar to Proposition 1) that the fuzzy support $F Z S\left(Z, \mu_{Z}\right)$ of a fuzzy set $\left(Z, \mu_{Z}\right)$ over the invariant set $(U, \cdot)$ is a finitely supported subset of $U$ with the property that $\operatorname{supp}\left(F Z S\left(Z, \mu_{Z}\right)\right) \subseteq \operatorname{supp}\left(\mu_{Z}\right)$. Moreover, in the particular case when the fuzzy support $F Z S\left(Z, \mu_{Z}\right)$ is finite, we have the following result that presents a relationship between the (fuzzy) support and the atomic support of a fuzzy set.

Proposition 2. Considering the fuzzy set $\left(Z, \mu_{Z}\right)$ over the invariant set $(U, \cdot)$, if $F Z S\left(Z, \mu_{Z}\right)$ is finite, then $\operatorname{supp}\left(F Z S\left(Z, \mu_{Z}\right)\right)=\operatorname{supp}\left(\mu_{Z}\right)$. Particularly, if $\left(Z, \mu_{Z}\right)$ is a fuzzy set over the invariant set $A$ of atoms and $F Z S\left(Z, \mu_{Z}\right)$ is finite, then we have $F Z S\left(Z, \mu_{Z}\right)=\operatorname{supp}\left(\mu_{Z}\right)$.

More generally, finitely supported functions from the set of atoms $A$ to a non-atomic $Z F$ set $Z$ (e.g., $Z$ can be the unit interval [0,1] have the following property which allows to connect our notion of support with the classical notion of algebraic support.

Theorem 1. Let $(Z, \diamond)$ be an infinite non-atomic $Z F$ set and $f: A \rightarrow Z$ a function.

1. If $f$ is finitely supported, then there is $z_{0} \in Z$ such that $\left\{a \in A \mid f(a) \neq z_{0}\right\}$ is finite.

2. If there is $z_{0} \in Z$ such that $\left\{a \in A \mid f(a) \neq z_{0}\right\}$ is finite, then $f$ is finitely supported and $\operatorname{supp}(f)=\left\{a \in A \mid f(a) \neq z_{0}\right\}$.

For the fuzzy sets over invariant sets, we define operations similarly to those in ZF.

Lemma 1. Let $\left(X, \mu_{X}\right)$ and $\left(Y, \mu_{Y}\right)$ be fuzzy sets over the invariant set $(U, \cdot)$.

1. Then $X \cup Y$ is finitely supported, and the function $x \mapsto \max \left[\mu_{X}(x), \mu_{Y}(x)\right]$ defined on $U$ is also finitely supported.

2. Then $X \cap Y$ is finitely supported and, furthermore, the function $x \mapsto \min \left[\mu_{X}(x), \mu_{Y}(x)\right]$ defined on $U$ is also finitely supported.

3. Both the complementary of $X$ (denoted by $C_{X}$ ) and the function $x \mapsto 1-\mu_{X}(x)$ defined on $U$ are finitely supported.

According to Lemma 1, the following definition is valid for fss.

Definition 4. Let $\left(X, \mu_{X}\right)$ and $\left(Y, \mu_{Y}\right)$ be two fuzzy sets over the invariant set $(U, \cdot)$.

1. The union of two fuzzy sets $X$ and $Y$ is a fuzzy set over the invariant set $U$ given by the finitely supported subset $X \cup Y$ of $U$ with the finitely supported membership function $\mu_{X \cup Y}: U \rightarrow$ $[0,1]$ defined by $\mu_{X \cup Y}(x)=\max \left[\mu_{X}(x), \mu_{Y}(x)\right]$ for all $x \in U$.

2. The intersection of two fuzzy sets $X$ and $Y$ is a fuzzy set over the invariant set $U$ given by the finitely supported subset $X \cap Y$ of $U$ with the finitely supported membership function $\mu_{X \cap Y}: U \rightarrow[0,1]$ defined by $\mu_{X \cap Y}(x)=\min \left[\mu_{X}(x), \mu_{Y}(x)\right]$ for all $x \in U$. 
3. The complement of a fuzzy set $X$ is a fuzzy set over the invariant set $U$ given by the finitely supported subset $C_{X}$ of $U$ together with the finitely supported membership function $\mu_{C_{X}}$ : $U \rightarrow[0,1]$ defined by $\mu_{C_{X}}(x)=1-\mu_{X}(x)$ for all $x \in U$.

Proposition 3. Let $\left(X, \mu_{X}\right)$ and $\left(Y, \mu_{Y}\right)$ be fuzzy sets over the invariant set $(U, \cdot)$. Then, we have the following relations:

1. $C_{\left(X, \mu_{X}\right) \cap\left(Y, \mu_{Y}\right)}=C_{\left(X, \mu_{X}\right)} \cup C_{\left(Y, \mu_{Y}\right)}$;

2. $C_{\left(X, \mu_{X}\right) \cup\left(Y, \mu_{Y}\right)}=C_{\left(X, \mu_{X}\right)} \cap C_{\left(Y, \mu_{Y}\right)}$.

Lemma 2. Let $\mathcal{F}=\left(Z_{i}, \mu_{Z_{i}}\right)_{i \in I}$ be a family of fuzzy sets over the invariant set $(U, \cdot)$ which is finitely supported as a subset of $\left(\wp_{f_{s}}(U) \times[0,1]_{f_{\mathcal{S}}}^{U}, \otimes\right)$. Then, $\cup_{i \in I} Z_{i}$ is finitely supported by $\operatorname{supp}(\mathcal{F})$, and the function $z \mapsto \underset{i \in I}{\vee}\left\{\mu_{Z_{i}}(z) \mid i \in I\right\}$ defined on $U$ is finitely supported by $\operatorname{supp}(\mathcal{F})$, where $\vee$ represents the notation for supremum (least upper bound).

Lemma 3. Let $\mathcal{F}=\left(Z_{i}, \mu_{Z_{i}}\right)_{i \in I}$ be a family of fuzzy sets over the invariant set $(U, \cdot)$ which is finitely supported as a subset of $\left(\wp_{f_{s}}(U) \times[0,1]_{f_{s}}^{U}, \otimes\right)$. Then, $\cap_{i \in I} Z_{i}$ is finitely supported by $\operatorname{supp}(\mathcal{F})$, and the function $z \mapsto \wedge\left\{\mu_{i \in I}(z) \mid i \in I\right\}$ defined on $U$ is also finitely supported by $\operatorname{supp}(\mathcal{F})$, where $\wedge$ represents the notation for infimum (greatest lower bound).

Due to Lemmas 2 and 3, the next definition is also valid for finitely supported structures.

Definition 5. Let $\mathcal{F}=\left(Z_{i}, \mu_{Z_{i}}\right)_{i \in I}$ be a family of fuzzy sets over the invariant set $(U, \cdot)$ which is finitely supported as a subset of $\left(\wp_{f_{S}}(U) \times[0,1]_{f_{\mathcal{S}}}^{U}, \otimes\right)$.

1. The arbitrary union of the fuzzy sets $\left(Z_{i}, \mu_{Z_{i}}\right)_{i \in I}$ is a fuzzy set over the invariant set $U$ represented by the finitely supported subset $\cup Z_{i}$ of $U$ together with the finitely supported function $\mu_{i \in I} Z_{i}: U \rightarrow[0,1]$ defined by $\mu_{i \in I} Z_{i}(z)=\bigvee_{i \in I}\left\{\mu_{Z_{i}}(z) \mid i \in I\right\}$.

2. The arbitrary intersection of the family of fuzzy sets $\left(Z_{i}, \mu_{Z_{i}}\right)_{i \in I}$ is a fuzzy set over the invariant set $U$ represented by the finitely supported subset $\cap Z_{i \in I}$ of $U$ together with the finitely supported function $\mu_{i \in I} Z_{i}: U \rightarrow[0,1]$ defined by $\mu_{i \in I} Z_{i}(z)=\wedge_{i \in I}\left\{\mu_{Z_{i}}(z) \mid i \in I\right\}$.

According to the extension principle in the classical theory of fuzzy sets, the domain of a function to be extended from crisp points in the universe $U$ to fuzzy sets in $U$ is allowed. More precisely, let $f: U \rightarrow V$ be a function from a crisp set $U$ to a crisp set $V$. Suppose that we have a given fuzzy set $Z$ in $U$, and want to determine a fuzzy set $Y$ in $V$ induced by $f$ (i.e., $Y=f(Z)$ ). In general, the membership function for $Y$ is defined by

$$
\mu_{Y}(y)=\underset{z \in f^{-1}(y)}{\vee} \mu_{Z}(z) \text {, where } y \in V \text { and } f^{-1}(y)=\{z \in U \mid f(z)=y\} \text {. }
$$

Theorem 2. Let $(U, \cdot)$ and $(V, \diamond)$ be two invariant sets, and consider a finitely supported function $f: U \rightarrow V$. If $\left(Z, \mu_{Z}\right)$ is a fuzzy set over the invariant set $(U, \cdot)$; then, $Y=f(Z)$ is a fuzzy set over the invariant set $(V, \diamond)$ with the finitely supported membership function $\mu_{Y}: V \rightarrow[0,1]$ defined as follows:

$$
\mu_{Y}(y)=\left\{\begin{array}{cl}
V_{z \in f^{-1}(y)} \mu_{Z}(z) & \text { for } y \in V, f^{-1}(y) \neq \varnothing \\
0 & \text { for } y \in V, f^{-1}(y)=\varnothing
\end{array} .\right.
$$

Moreover, we have supp $(Y) \subseteq \operatorname{supp}(f) \cup \operatorname{supp}(Z)$, and $\operatorname{supp}\left(\mu_{Y}\right) \subseteq \operatorname{supp}(f) \cup \operatorname{supp}\left(\mu_{Z}\right)$.

Theorem 3. Let $\left(U_{1}, \cdot 1\right), \ldots,\left(U_{n}, \cdot n\right)$ and $(V, \diamond)$ be invariant sets, and a finitely supported function $f: U_{1} \times \ldots \times U_{n} \rightarrow V$. If $\left(Z_{i}, \mu_{Z_{i}}\right)$ is a fuzzy set over the invariant set $\left(U_{i}, v_{i}\right)$ for all $i \in\{1, \ldots n\}$, then $Y=f\left(Z_{1} \times \ldots \times Z_{n}\right)$ is a fuzzy set over the invariant set $(V, \diamond)$ with the finitely supported membership function $\mu_{Y}: V \rightarrow[0,1]$ defined as follows: 


$$
\begin{gathered}
\mu_{Y}(y)=\underset{y=f\left(z_{1}, \ldots, z_{n}\right)}{\vee}\left[\min \left(\mu_{Z_{1}}\left(z_{1}\right), \ldots, \mu_{Z_{n}}\left(z_{n}\right)\right)\right] \\
\text { for } y \in V \text { and } f^{-1}(y) \neq \varnothing ; \\
\mu_{Y}(y)=0 \quad \text { for } y \in V \text { and } f^{-1}(y)=\varnothing .
\end{gathered}
$$

Moreover, we have that $\operatorname{supp}(Y) \subseteq \operatorname{supp}(f) \cup \operatorname{supp}\left(Z_{1}\right) \cup \ldots \cup \operatorname{supp}\left(Z_{n}\right)$, and $\operatorname{supp}\left(\mu_{Y}\right) \subseteq$ $\operatorname{supp}(f) \cup \operatorname{supp}\left(\mu_{Z_{1}}\right) \cup \ldots \cup \operatorname{supp}\left(\mu_{Z_{n}}\right)$.

\section{Degree of Membership Association for Invariant Sets}

A fuzzy set is an element of the invariant set $\left(\wp_{f_{s}}(U) \times[0,1]_{f_{s}}^{U}, \diamond\right)$. In such a Cartesian pair, we have not yet required the existence of an fss association between the crisp finitely supported subset of $U$ and the related finitely supported function in $[0,1]_{f s}^{U}$; such a firm fss association should itself preserve the finite support requirement, and for the previous results, such a condition was not mandatory. We now analyse the case when such an relationship between $\wp_{f_{s}}(U)$ and $[0,1]_{f s}^{U}$ is defined.

Definition 6. Let us consider the invariant set $(U, \cdot)$. A fss degree of membership association over $U$ is an equivariant binary relation $R$ on $\wp_{f s}(U)$ and $[0,1]_{f s}^{U}$, i.e., an equivariant (i.e., empty supported) subset $R$ of $\wp_{f s}(U) \times[0,1]_{f_{s}}^{U}$.

Lemma 4. Let $Y$ be a finitely supported subset of an invariant set $(U, \cdot)$, and $\chi_{Y}$ be the characteristic function on $Y$, i.e.,

$$
\chi_{Y}(y) \stackrel{\text { def }}{=}\left\{\begin{array}{ll}
1 & \text { for } y \in Y \\
0 & \text { for } y \in U \backslash Y
\end{array} .\right.
$$

Then $\chi_{Y}: U \rightarrow[0,1]$ is a finitely supported function for any $Y \in \wp_{f s}(U)$, and the function $Z \mapsto \chi_{Z}$ defined on $\wp_{f s}(U)$ is equivariant.

Let $\operatorname{algsup}(f) \stackrel{\text { def }}{=}\{z \in U \mid f(z)>0\}$ be the algebraic support of $f$, where $(U, \cdot)$ is an invariant set and $f: U \rightarrow[0,1]$ a finitely supported function.

Lemma 5. The algebraic support algsup $(f)$ is a finitely supported subset of $U$. Moreover, the function $f \mapsto \operatorname{algsup}(f)$ defined on $[0,1]_{f s}^{U}$ is equivariant.

We provide some examples of fss degree of membership associations.

Example 1. Let $(U, \cdot)$ be an invariant set.

1. We define $R=\left\{\left(Y, \chi_{Y}\right) \mid Y \in \wp_{f_{s}}(U)\right\}$, where $\chi_{Y}$ represents the characteristic function of $Y$. According to Lemma $4, R$ is equivariant, and so $R$ is a fss degree of membership association over $U$.

2. We define $R=\left\{(\operatorname{algsup}(f), f) \mid f \in[0,1]_{f s}^{U}\right\}$, where algsup $(f)$ represents the algebraic support of $f$. According to Lemma $5, R$ is equivariant, and so $R$ is a fss degree of membership association over $U$.

Definition 7. Let $(U, \cdot)$ be an invariant set. A full fss degree of membership association over the invariant set $U$ is an equivariant binary relation $F$ on $\wp_{f s}(U)$ and $[0,1]_{f s}^{U}$ (i.e., an equivariant subset $F$ of the invariant set $\left.\wp_{f_{s}}(U) \times[0,1]_{f s}^{U}\right)$ satisfying the following conditions:

1. $F$ is a left-total binary relation; namely, for any finitely supported subset $Z$ of $U$, a finitely supported function $f: U \rightarrow[0,1]$ called F-degree of membership function of $Z$ such that $(Z, f) \in F$.

2. $F$ is an onto binary relation; namely, for every finitely supported function $f: U \rightarrow[0,1]$, $Z \in \wp_{f s}(U)$ such that $(Z, f) \in F$. 
The conditions in Definition 7 correspond to our intuition of how a full fss degree of membership association over an invariant set should be defined.

1. The first condition in Definition 7 means that for each element in $\wp_{f s}(U)$, we should find at least one associated fss degree of membership function which models the degree of membership in $X$ for each element in $U$ (at least the characteristic function of $X$ is such a finitely supported function).

2. The second condition in Definition 7 means that any element of $[0,1]_{f_{S}}^{U}$ should be a fss degree of membership function associated with a certain element of $\wp_{f_{s}}(U)$. For each $f \in[0,1]_{f_{S}}^{U}$, we could consider that $f$ is associated to at least its algebraic support.

Example 2. Let $(U, \cdot)$ be an invariant set.

Let $F=\left\{\left(Y, \chi_{Y}\right) \mid Y \in \wp_{f_{S}}(U)\right\} \cup\left\{(\operatorname{algsup}(f), f) \mid f \in[0,1]_{f s}^{U}\right\}$, where $\chi_{Y}$ represents the characteristic function of $Y$ and algsup $(f)$ represents the algebraic support of $f$. Then $F$ is equivariant. Furthermore, $F$ is a full fss degree of membership association over $U$.

\section{Proposition 4.}

1. Let $(U, \cdot)$ be an invariant set such that there is a fss degree of membership association $F$ over it. Then the set of all F-degree of membership functions of $U$, i.e., $\wp_{\text {fuzzy }}^{F}(U) \stackrel{\text { def }}{=}\{f \in$ $\left.[0,1]_{f_{S}}^{U} \mid \exists Z \in \wp_{f_{S}}(U),(Z, f) \in F\right\}=\operatorname{Im}(F)$ is an equivariant subset of $\left([0,1]_{f_{S}}^{U}, \widetilde{*}\right)$, where $\approx$ is the standard $S_{A}$-action on $[0,1]_{f s}^{U}$.

2. Let $(U, \cdot)$ be an invariant set such that there is a full $f_{s s}$ degree of membership association $F$ over it. Then the set of all F-degrees of membership functions defined on $U$, namely, $\wp_{f u z z y}^{F}(U)$, is an invariant set that coincides with $\left([0,1]_{f_{s}}^{U}, \widetilde{\star}\right)$.

Theorem 4. Let $(U, \cdot)$ be an invariant set such that there is a full fss degree of membership association F over the invariant set $U$. Then $\left(\wp_{f u z z y}^{F}(U), \widetilde{\star}, \sqsubseteq\right)$ is an invariant complete lattice, where $\sqsubseteq$ is an equivariant order relation on $\wp_{\text {fuzzy }}^{F}(U)=\left\{f \in[0,1]_{f s}^{U} \mid \exists Z \in \wp_{f_{s}}(U),(Z, f) \in\right.$ $F\}$ defined by: $f \sqsubseteq g$ is and only if $f(x) \leq g(x)$ for all $x \in U$.

To prove Theorem 4 , when $\mathcal{F}=\left(f_{i}\right)_{i \in I}$ is a finitely supported family of elements from $[0,1]_{f_{s}}^{U}$, we define $\underset{i \in I}{\sqcup} f_{i}: U \rightarrow[0,1]$ by $\left.\underset{i \in I}{\sqcup} f_{i}\right)(x)=\underset{i \in I}{\operatorname{supremum}}\left\{f_{i}(x) \mid i \in I\right\}$ for all $x \in U$, where by supremum we denoted the least upper bounds in the set of real numbers. Using the fact that, whenever $\pi \in \operatorname{Fix}(\operatorname{supp}(\mathcal{F}))$, for any $i \in I$ there is a unique $j \in I$ such that $f_{i}=\pi^{-1} \widetilde{\star} f_{j}$ (where $\widetilde{\star}$ is the $S_{A}$-action on $[0,1]_{f_{s}}^{U}$ ), we obtain that for each $i \in I$ there is a unique $j \in I$ such that $f_{i}(x)=f_{j}(\pi \cdot x)$ for all $x \in U$. Then we concluded that $\operatorname{supremum}\left\{f_{i}(\pi \cdot x) \mid i \in I\right\}=\underset{i \in I}{\operatorname{supremum}}\left\{f_{i}(x) \mid i \in I\right\}$ for all $x \in U$ and all $\pi \in \operatorname{Fix}(\operatorname{supp}(\mathcal{F}))$, from which we obtained that $\operatorname{supp}(\mathcal{F})$ supports $\sqcup f_{i \in I}$, which means $\sqcup f_{i \in I}$ is the least upper bound of $\mathcal{F}$ in $[0,1]_{f s}^{U}=\wp_{f u z z y}^{F}(U)$ (for the last relation we used Proposition 4(2)).

Several properties of $\left(\wp_{f u z z y}^{F}(U), \widetilde{\star}, \sqsubseteq\right)$ are obtained from the general properties of invariant complete lattices [6].

Corollary 1. Let $(U, \cdot)$ be an invariant set such that there is a full fss degree of membership association $F$ over $U$.

1. Let $\varphi: \wp_{\text {fuzzy }}^{F}(U) \rightarrow \wp_{\text {fuzzy }}^{F}(U)$ be a finitely supported order preserving function over $\left(\wp_{f u z z y}^{F}(U), \widetilde{\star}, \sqsubseteq\right)$. Then there is a greatest $f \in \wp_{f u z z y}^{F}(U)$ such that $\varphi(f)=f$, as well as a least $g \in \wp_{\text {fuzzy }}^{F}(U)$ such that $\varphi(g)=g$. 
2. Let $\varphi: \wp_{\text {fuzzy }}^{F}(U) \rightarrow \wp_{\text {fuzzy }}^{F}(U)$ be a finitely supported order-preserving function over $\left(\wp_{\text {fuzzy }}^{F}(U), \widetilde{\star}, \sqsubseteq\right)$. Let $P$ be the set of fixed points of $\varphi$. Then $(P, \widetilde{\star}, \sqsubseteq)$ is a finitely supported (by $\operatorname{supp}(\varphi)$ ) complete lattice.

It is worth noting that we obtain properties that cannot be obtained with standard fuzzy techniques in ZF. For instance, there exist lattices that are invariant complete, but fail to be complete in the ZF framework. A related example is presented in [11], where we proved that the set of those subsets of $A$ which are either finite or cofinite is an invariant complete lattice (with the classical inclusion order), but it fails to be a complete lattice in ZF. Another such example is presented in Proposition 4.

Proposition 5. Let us assume that there is a full fss degree of membership association $F$ over the invariant set of atoms $A$. Then $\left(\wp_{\text {fuzzy }}^{F}(A), \widetilde{\star}, \sqsubseteq\right)$ is an invariant complete lattice, but it fails to be a complete lattice in $\mathrm{ZF}$ framework when $A$ is considered as a set in $\mathrm{ZF}$.

In order to prove Proposition 5, we considered $P$ to be a fixed ZF simultaneously infinite and coinfinite subset of $A$. For each $a \in A$ we defined $\varphi_{a}: A \rightarrow[0,1]$ by $\varphi_{a}(b)=$ $\left\{\begin{array}{ll}1 & \text { for } b=a \\ 0 & \text { for } b \in A \backslash\{a\}\end{array}\right.$. Any function $\varphi_{a}$ is supported by $\operatorname{supp}(a)$. Moreover, we proved that the function $j: A \rightarrow[0,1]_{f_{s}}^{A}$ defined by $j(a)=\varphi_{a}$ for all $a \in A$ is equivariant. We considered the infinite family $\mathcal{F}$ from $[0,1]_{f s}^{A}$ defined by $\mathcal{F}=\left\{\varphi_{a} \mid a \in P\right\}$. The only possible least upper bound of $\mathcal{F}$ would have been the function $\psi: A \rightarrow[0,1]$ defined by $\psi(x)=\left\{\begin{array}{ll}1 & \text { for } x \in P \\ 0 & \text { for } x \in A \backslash P\end{array}\right.$. Since $P$ is not finitely supported, it followed that $\psi$ is not finitely supported, and so $\mathcal{F}$ does not have a least upper bound in $[0,1]_{f s}^{A}$.

Since the construction of $\wp_{f u z z y}^{F}(A)=[0,1]_{f s}^{A}$ makes sense in $\mathrm{ZF}$, the previous result shows that $\wp_{f u z z y}^{F}(A)$ is a lattice which is not complete in $\mathrm{ZF}$, but is the only invariantcomplete. This aspect emphasises one benefit of this approach: even though we have only a refined form of completeness (namely, the invariant completeness) in ZF for $\wp_{f u z z y}^{F}(A)$, we can provide new properties of $\wp_{f u z z y}^{F}(A)$ derived from the general properties of the invariant complete lattices (presented in [6]).

Invariant monoids were introduced in [12] as invariant sets equipped with equivariant internal monoid laws. More exactly, $(M,+, \cdot)$ is an invariant monoid if $(M, \cdot)$ is an invariant set and $(M,+, 0)$ is a monoid having the properties that $\pi \cdot(x+y)=(\pi \cdot x)+(\pi \cdot y)$, $\pi \cdot 0=0$ for all $x, y \in M$ and $\pi \in S_{A}$.

Theorem 5. Let $(U, \cdot)$ be an invariant set such that there is a full fss degree of membership association $F$ over it. Then $\wp_{\text {fuzzy }}^{F}(U)$ can be organised as an invariant monoid in the following two forms:

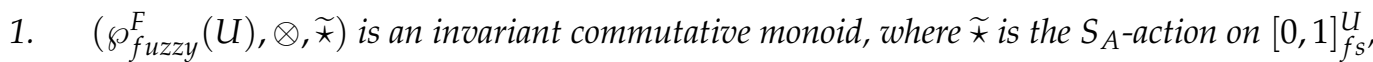
and $f \otimes g: U \rightarrow[0,1]$ is defined by the relation $(f \otimes g)(x)=f(x) g(x)$ for all $x \in U$. The neutral element is the equivariant function $1_{U}: U \rightarrow[0,1]$ defined by $1_{U}(x)=1$ for all $x \in U$.

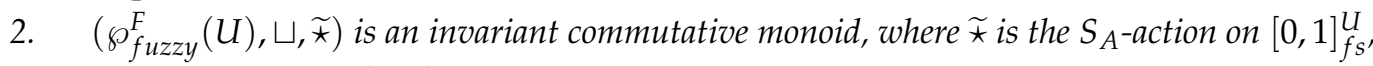
and $f \sqcup g: U \rightarrow[0,1]$ is defined by the relation $(f \sqcup g)(x)=\operatorname{supremum}\{f(x), g(x)\}$ for all $x \in U$. The neutral element is the equivariant function $0_{U}: U \rightarrow[0,1]$ defined by $0_{U}(x)=0$ for all $x \in U$.

The general properties of invariant monoids presented in [12] lead to new properties of $\wp_{f u z z y}^{F}(U)$ (equipped with one of the two internal laws defined in Theorem 5). Some of them are related to the invariant isomorphism theorems, to invariant universality 
theorems or to Cayley monoids theorem. We present here an fss Cayley-type embedding theorem for $\wp_{\text {fuzzy }}^{F}(U)$ which follows from Theorem 7 in [12].

Theorem 6. Let $(U, \cdot)$ be an invariant set with the property that there is a full fss degree of membership association $F$ over it. Then there is an equivariant isomorphism between $\wp_{\text {fuzzy }}^{F}(U)$ and an invariant submonoid of the invariant monoid formed by the finitely supported elements of $\wp_{f u z z y}^{F}(U)^{\wp_{f u z z y}^{F}(U)}$.

The universality properties for invariant monoids [12] allow us to establish connectivity results between the set of all fuzzy sets over an invariant set $U$, the free monoid over $U$ and the set of all extended multisets over $U$.

Let $(U, \cdot)$ be an invariant set such that there is a full fss degree of membership association $F$ over it. Let $\mathbb{N}_{\text {ext }}(U)$ be the set of all extended multisets over $U$ (defined as functions $f: U \rightarrow \mathbb{N}$ with finite algebraic supports, which are proved to be finitely supported by their algebraic supports, where $\mathbb{N}$ is the set of all positive integers). Then, $\mathbb{N}_{\text {ext }}(U)$ endowed with the classical pointwise sum of extended multisets is an invariant monoid with the same $S_{A}$-action as $\mathbb{N}_{f_{S}}^{U}$. If $U^{*}$ is the free monoid on $U$, then $U^{*}$ endowed with the classical juxtaposition of words is an invariant monoid with the $S_{A}$ action $\diamond$ defined by $\pi \diamond x_{1} \ldots x_{n}=\left(\pi \cdot x_{1}\right) \ldots\left(\pi \cdot x_{n}\right)$ for all $\pi \in S_{A}, x_{1} \ldots x_{n} \in U^{*} \backslash\{1\}$, and $\pi \diamond 1=1$ for all $\pi \in S_{A}$ (where 1 is the empty word).

Theorem 7. Let $U, V$ be invariant sets with the property that there is a full fss degree of membership association with $V$. Let $j: U \rightarrow \mathbb{N}_{\text {ext }}(U)$ be the function which maps each $x \in U$ into the characteristic function $\chi_{\{x\}}$. If $\phi: U \rightarrow \wp_{\text {fuzzy }}^{F}(V)$ is an arbitrary finitely supported function, then there is a unique finitely supported homomorphism of invariant commutative monoids $\psi: \mathbb{N}_{\text {ext }}(U) \rightarrow \wp_{\text {fuzzy }}^{F}(V)$ with $\psi \circ j=\phi$, i.e., $\psi\left(\chi_{\{x\}}\right)=\phi(x)$ for all $x \in U$. Furthermore, $\operatorname{supp}(\psi) \subseteq \operatorname{supp}(\phi)$.

Theorem 8. Let $U, V$ be invariant sets with the property that there is a full fss degree of membership association with $V$. Let $i: U \rightarrow U^{*}$ be the standard inclusion of $U$ into $U^{*}$ which maps each element $x \in U$ into the word $x$. If $\phi: U \rightarrow \wp_{\text {fuzzy }}^{F}(V)$ is an arbitrary finitely supported function, then there is a unique finitely supported homomorphism of invariant monoids $\psi: U^{*} \rightarrow \wp_{\text {fuzzy }}^{F}(V)$ with $\psi \circ i=\phi$. Furthermore, $\sup p(\psi) \subseteq \operatorname{supp}(\phi)$.

The following isomorphism theorem can be proved from the general properties of invariant monoids [5]. For its corollaries, we involve Theorem 5 and the fact that the function $f \mapsto F Z S(X, f)$ is an equivariant homomorphism between $(F A S(X), \sqcup, \widetilde{\star})$ and $\left(\wp_{\text {fin }}(X), \cup, \star\right)$ with the notations in Corollary 3.

Theorem 9. Let $\left(M,+_{M}, \cdot\right)$ and $\left(N,+_{N}, \diamond\right)$ be invariant monoids and let $f: M \rightarrow N$ be an equivariant homomorphism. On $M$ we define the relation $\sim_{f}$ by: $m_{1} \sim_{f} m_{2}$ if $f\left(m_{1}\right)=f\left(m_{2}\right)$. Then $\sim_{f}$ is an equivariant equivalence relation and there is an equivariant isomorphism $\varphi$ between the invariant factor monoid $\left(M / \sim_{f},+, \star\right)$ and the invariant monoid $\operatorname{Im}(f)$, defined by $\varphi([m])=$ $f(m)$ for all $m \in M$, whereby $[m]$ we denoted the equivalence class of $m$ modulo $\sim_{f}$; the internal law + is defined by $:[m]+\left[m^{\prime}\right]=\left[m+{ }_{M} m^{\prime}\right]$ for all $[m],\left[m^{\prime}\right] \in M / \sim_{f}$ and the $S_{A}$-action $\star$ is defined by $\pi \star[m]=[\pi \cdot m]$ for all $[m] \in M / \sim_{f}$.

Corollary 2. Let $(X, \cdot)$ be an invariant set with the property that there is a full fss degree of membership association with $X$, and let $\left(N,+_{N}, \diamond_{N}\right)$ be an invariant monoid. Let $\psi: \wp_{\text {fuzzy }}^{F}(X) \rightarrow N$ be an equivariant homomorphism. On $\wp_{\text {fuzzy }}^{F}(X)$ (equipped with the internal laws $\otimes$ or $\sqcup$ ), we define the relation $\sim_{\psi}$ by: $f_{1} \sim_{\psi} f_{2}$ if $\psi\left(f_{1}\right)=\psi\left(f_{2}\right)$. Then $\sim_{\psi}$ is an equivariant equivalence relation and there is an equivariant isomorphism $\varphi$ between the invariant monoid $\wp_{\text {fuzzy }}^{F}(X) / \sim_{\psi}$ 
and the invariant monoid $\operatorname{Im}(\psi)$, defined by $\varphi([f])=\psi(f)$ for all $f \in \wp_{\text {fuzzy }}^{F}(X)$, whereby $[f]$ we denoted the equivalence class of $f$ modulo $\sim_{\psi}$.

Corollary 3. Let $(X, \cdot)$ be an invariant set with the property that there is a full fss degree of membership association with $X$. Let $F A S(X)=\{f: X \rightarrow[0,1] \mid F Z S(X, f)$ is finite $\}$. On FAS $(X)$ we define the relation $\sim$ by: $f \sim g$ if and only if $F Z S(X, f)=F Z S(X, f)$. Then FAS $(X)$ is an equivariant submonoid of $\left(\wp_{\text {fuzzy }}^{F}(X), \sqcup, \widetilde{\star}\right)$ and there is an equivariant isomorphism $\varphi$ between the invariant factor monoid $F A S(X) / \sim$ and the invariant monoid $\left(\wp_{\text {fin }}(X), \cup, \star\right)$ defined by $\varphi([f])=F Z S(X, f)$ for all $f \in F A S(X)$, whereby $[f]$ we denoted the family of functions from $X$ to $[0,1]$ having the same algebraic support as $f$.

\section{L-Fuzzy Sets and Invariant Complete Lattices}

We present the notion of $L$-fuzzy set and several fixed point properties in this framework. By now on, we implicitly assume that the invariant sets we involve are endowed with a full fss degree of membership associations. Therefore, for an invariant algebraic structure $P$, the $P$-fuzzy sets over an invariant set $U$ will be defined as finitely supported functions from $U$ to $P$.

Definition 8. Let $(L, \cdot, \sqsubseteq)$ be an invariant complete lattice and $(U, \diamond)$ an invariant set.

- An L-fuzzy set over $U$ is a finitely supported function $\mu: U \rightarrow L$.

- $\quad$ The algebraic support of a function $f: U \rightarrow L$ is the crisp set FZS $(U, f)=\{x \in$ $U \mid 0 \sqsubset f(x)\}$.

\section{Example 3.}

- $\quad$ Let $U$ be an invariant set. The function $f: U \rightarrow \wp_{f_{s}}(A)$ defined by $f(x)=\operatorname{supp}(x)$ for all $x \in U$ is an equivariant $L$-fuzzy set over $U$. This is because $\wp_{f s}(A)$ is an invariant complete lattice and, for all $\pi \in S_{A}$, we have $\operatorname{supp}(\pi \cdot x)=\pi(\operatorname{supp}(x))=\pi \star \operatorname{supp}(x)$ for all $x \in X$.

- Let $(X, \cdot)$ be an invariant set. Let $\varphi:[0,1]_{f s}^{X} \rightarrow \wp_{f s}(X), \varphi(f)=F Z S(X, f)$. For $\pi \in S_{A}$ we verify that $\pi \star F Z S(X, f)=F Z S(X, \pi \widetilde{\star} f)$ for all $f \in[0,1]_{f s}^{X}$. Fix $f$ and let $z \in \pi \star F Z S(X, f)$. Then $z=\pi \cdot x$ with $x \in F Z S(X, f)$, and hence $(\pi \approx f)(z)=$ $f\left(\pi^{-1} \cdot z\right)=f(x)>0$, i.e., $z \in F Z S(X, \pi \widetilde{\star} f)$. Conversely, let $z \in F Z S(X, \pi \widetilde{\star} f)$. It follows that $f\left(\pi^{-1} \cdot z\right)>0$. Thus, $z=\pi \cdot\left(\pi^{-1} \cdot z\right)$ with $\pi^{-1} \cdot z \in F Z S(X, f)$, and so $z \in \pi \star F Z S(X, f)$. Thus, since $\wp_{f s}(X)$ is an invariant complete lattice, we have that $\varphi$ is an equivariant $L$-fuzzy set over $[0,1]_{f_{s}}^{X}$.

Theorem 10. Let $(L, \cdot, \sqsubseteq)$ be an invariant complete lattice and $(U, \diamond)$ an invariant set. Any function $f: U \rightarrow L$ has the following properties:

1. If $f$ is an $L$-fuzzy set over $U$, then $F Z S(U, f)$ is finitely supported, and:

- $\operatorname{supp}(F Z S(U, f)) \subseteq \operatorname{supp}(f)$;

- $\operatorname{supp}(f(F Z S(U, f))) \subseteq \operatorname{supp}(F Z S(U, f)) \cup \operatorname{supp}(f)$;

2. If the algebraic support of $f$ is finite, then $f$ is an L-fuzzy set over $U$ (i.e., $f$ is finitely supported) and $\operatorname{supp}(f) \subseteq \operatorname{supp}(F Z S(U, f)) \cup \operatorname{supp}(f(F Z S(U, f)))$.

3. If the algebraic support of $f$ is finite, then $\operatorname{supp}(f(F Z S(U, f))) \backslash \operatorname{supp}(F Z S(U, f))=$ $\operatorname{supp}(f) \backslash \operatorname{supp}(F Z S(U, f))$.

The L-fuzzy sets are characterised by the following property.

Theorem 11. Let $(U, \diamond)$ be an invariant set and $(L, \cdot, \sqsubseteq)$ an invariant complete lattice.

- The family of those finitely supported functions $f: U \rightarrow L$ (i.e., the family $L_{f s}^{U}$ of all $L$-fuzzy sets over the invariant set $U)$ is an invariant complete lattice with the order relation $\leq$ defined by $f \leq g$ if and only if $f(x) \sqsubseteq g(x)$ for all $x \in U$. 
- $\quad$ Furthermore, if $\mathcal{F}=\left(f_{i}\right)_{i \in I}$ is a finitely supported family of L-fuzzy sets over the invariant set $U$, its least upper bound with respect to $\leq$ is $\underset{i \in I}{\vee} f_{i}: U \rightarrow$ L defined by $\left.\underset{i \in I}{\vee} f_{i}\right)(x)=$ $\sqcup\left\{f_{i}(x) \mid i \in I\right\}$ for all $x \in U$, whereby $\sqcup$ we denoted least upper bounds in $L$ with respect to $\sqsubseteq$.

The requirement that $L$ is invariant complete in Theorem 11 is necessary. For example, let us fix an element $a \in A$; the family $\left(f_{n}\right)_{n \in \mathbb{N}}$ of functions from $A$ to $\mathbb{N}$ defined by $f_{i}(b)=\left\{\begin{array}{ll}i & \text { for } b=a \\ 0 & \text { for } b \in A \backslash\{a\}\end{array}\right.$ for all $i \in \mathbb{N}$ is finitely supported (each $f_{i}$ is supported by the same set $\{a\})$, but it does not have a supremum modulo $\sqsubseteq$.

It is worth noting that some ZF structures are not finitely supported. The family of finitely supported functions from $U$ to $L$ makes sense in $Z F$, but it is an invariant complete lattice and not a fully ZF complete lattice in respect with all atomic sets.

According to Theorem 11, the following fixed point results can provide properties of finitely supported L-fuzzy sets over an invariant set. We chose to present the results in the general case, making them also applicable for other finitely supported structures (not only for finitely supported $L$-fuzzy sets).

In this section, we present fixed point theorems of Tarski's type in the framework of finitely supported structures. Tarski's theorem plays an important role in the theory of abstract interpretation of programming languages reformulated in the world of finitely supported structures [11].

Theorem 12 (Strong Tarski Theorem for fss). Let $(L, \cdot, \sqsubseteq)$ be an invariant complete lattice and $f: L \rightarrow L$ a finitely supported, order-preserving function. Let $F$ be the set of all fixed points of $f$. Then $(F, \cdot, \sqsubseteq)$ is itself a non-empty, finitely supported (by supp $(f))$, complete lattice.

In terms of $L$-fuzzy sets, this result states that, if $(L, \cdot, \sqsubseteq)$ is an invariant complete lattice and $f$ is an order-preserving $L$-fuzzy set over the invariant set $L$, then the set of fixed points of $f$ is itself a non-empty finitely supported (by supp $(f)$ ) complete lattice.

Corollary 4. Let $(L, \cdot, \sqsubseteq)$ be an invariant complete lattice and $f: L \rightarrow L$ a finitely supported, order-preserving function. Then $f$ has a least-fixed point defined as $\sqcap\{x \in L \mid f(x) \sqsubseteq x\}$ and a greatest-fixed point defined as $\sqcup\{x \in L \mid x \sqsubseteq f(x)\}$, which are both supported by supp $(f)$.

Corollary 5. Let $(L, \cdot, \sqsubseteq)$ be an invariant complete lattice and $f: L \rightarrow L$ an equivariant orderpreserving function. Let $F$ be the set of all fixed points of $f$. Then $(F, \cdot, \sqsubseteq)$ is itself an invariant complete lattice.

According to Theorem 13, Tarski's fixed-point theorem can be applied for finitely supported self-functions on the family of those finitely supported subsets of an invariant set [9].

Theorem 13. If $(X, \cdot)$ is an invariant set, then $\left(\wp_{f_{s}}(X), \star, \subseteq\right)$ is an invariant complete lattice.

Theorem 12 can be extended. We were able to prove the existence of fixed points of a finitely supported, order preserving self-function on an invariant partially ordered set, by imposing the existence condition of least upper bounds only for those uniformly supported subsets of the invariant, partially ordered set, and not for all finitely supported subsets of the related invariant, partially ordered set [10].

Theorem 14. Let $(X, \cdot, \sqsubseteq)$ be a non-empty invariant partially ordered set having the additional property that every uniformly supported subset of $X$ has a least upper bound. Let $f: X \rightarrow X$ be a finitely supported, order-preserving function with the property that there is $x_{0} \in X$ such that $x_{0} \sqsubseteq f\left(x_{0}\right)$. Then there is $u \in X$ with $x_{0} \sqsubseteq u$ such that $f(u)=u$. 
From Theorems 11 and 12, we obtain the following fixed point result for $L$-fuzzy sets over invariant sets.

Theorem 15. Let $(U, \diamond)$ be an invariant set, $(L, \cdot, \sqsubseteq)$ an invariant complete lattice and $\varphi: L_{f_{s}}^{U} \rightarrow$ $L_{f s}^{U}$ a finitely supported, order-preserving function over $L_{f s}^{U}$. Let $F$ be the set of fixed points of $\varphi$. Then $(F, \widetilde{\star}, \leq)$ is a non-empty, finitely supported (by supp $(f)$ ) complete lattice, where $\widetilde{\star}$ is the induced $S_{A^{-a c t i o n}}$ on the function space $L_{f_{s^{\prime}}}^{U}$ and $\leq$ is the order relation of the family $L_{f_{s}}^{U}$ of all $L$-fuzzy sets over the invariant set $U$ defined by $f \leq g$ if and only if $f(x) \sqsubseteq g(x)$ for all $x \in U$.

\section{Fuzzy Subgroups of an Invariant Group}

Rosenfeld introduced the notion of a fuzzy group and proved that many concepts of group theory can naturally be extended in order to develop the theory of fuzzy groups [13]. A survey of the development of fuzzy group theory can be found in [14].

Let us recall some results of the classical Zermelo-Fraenkel theory of fuzzy groups.

Definition 9. Let $(G, \cdot 1)$ be a group. On the family $\{v \mid v: G \rightarrow[0,1]\}$ of all fuzzy sets on $G$ we define a partial order relation $\sqsubseteq$, called fuzzy sets inclusion by $\eta \sqsubseteq \mu$ if and only if $\eta(x) \leq \mu(x)$ for all $x \in G$.

Definition 10. Let $(G, \cdot 1)$ be a group. A fuzzy set $\eta$ over the group $G$ (i.e., a function $\eta: G \rightarrow$ $[0,1])$ is called fuzzy subgroup of $G$ if the following conditions are satisfied:

- $\quad \eta(x \cdot y) \geq \min \{\eta(x), \eta(y)\}$ for all $x, y \in G$;

- $\eta\left(x^{-1}\right) \geq \eta(x)$ for all $x \in G$.

Definition 11. Let $(G, \cdot 1)$ be a group. A fuzzy subgroup $\mu$ of $G$ that satisfies the additional condition $\mu(x \cdot y)=\mu(y \cdot x)$ for all $x, y \in G$ is called a fuzzy normal subgroup of $G$.

Theorem 16. Let $(G, \cdot 1)$ be a group.

- The set $F L(G)$ formed by all fuzzy subgroups of $G$ is a complete lattice with respect to fuzzy sets inclusion.

- $\quad$ The set $F N(G)$ formed by all fuzzy normal subgroups of $G$ is a modular lattice with respect to fuzzy sets inclusion.

We translate the above results in the framework of finitely supported structures, proving their consistency within the new framework.

Definition 12. An invariant group is a triple $(G, \cdot, \diamond)$ with the property that the following conditions are satisfied:

- $G$ is a group with the internal law :;

- $G$ is a non-trivial invariant set with the $S_{A}$-action $\diamond$;

- for each $\pi \in S_{A}$ and each $x, y \in G$ we have $\pi \diamond(x \cdot y)=(\pi \diamond x) \cdot(\pi \diamond y)$, meaning that the internal law on $G$ is equivariant.

Proposition 6. $(G, \cdot, \diamond)$ be an invariant group. We have the following properties:

1. $\pi \diamond e=e$ for all $\pi \in S_{A}$, where $e$ is the neutral element of $G$.

2. $\pi \diamond x^{-1}=(\pi \diamond x)^{-1}$ for all $\pi \in S_{A}$ and $x \in G$.

We provide the following examples of invariant groups.

\section{Example 4.}

1. The group $\left(S_{A}, 0, \cdot\right)$ is an invariant group, where $\circ$ is the composition of permutations and $\cdot$ is the $S_{A}$-action on $S_{A}$ defined by $\pi \cdot \sigma:=\pi \circ \sigma \circ \pi^{-1}$ for all $\pi, \sigma \in S_{A}$. Since the composition 
of functions is associative, it is easy to verify that $\pi \cdot(\sigma \circ \tau)=(\pi \cdot \sigma) \circ(\pi \cdot \tau)$ for all $\pi, \sigma, \tau \in S_{A}$.

2. The free group $(F(X), \mathrm{T}, \tilde{\star})$ over an invariant set $(X, \diamond)$ (formed by those equivalence classes $[w]$ of words $w$, where two words are in the same equivalence class if one can be obtained from another by repeatedly inserting or cancelling terms of the form $u^{-1} u$ or $u u^{-1}$ for $\left.u \in X\right)$ is an invariant group, where $\widetilde{\star}: S_{A} \times F(X) \rightarrow F(X)$ is defined by $\pi \widetilde{\star}\left[x_{1}^{\varepsilon_{1}} x_{2}^{\varepsilon_{2}} \ldots x_{l}^{\varepsilon_{l}}\right]=[(\pi \diamond$ $\left.\left.x_{1}\right)^{\varepsilon_{1}} \ldots\left(\pi \diamond x_{l}\right)^{\varepsilon_{l}}\right]$, and $\left[x_{1}^{\varepsilon_{1}} x_{2}^{\varepsilon_{2}} \ldots x_{n}^{\varepsilon_{n}}\right] \mathrm{T}\left[y_{1}^{\delta_{1}} y_{2}^{\delta_{2}} \ldots y_{m}^{\delta_{m}}\right]=\left[x_{1}^{\varepsilon_{1}} x_{2}^{\varepsilon_{2}} \ldots x_{n}^{\varepsilon_{n}} y_{1}^{\delta_{1}} y_{2}^{\delta_{2}} \ldots y_{m}^{\delta_{m}}\right]$.

3. Given an invariant set $(X, \diamond)$, any function $f: X \rightarrow \mathbb{Z}$ (where $\mathbb{Z}$ is the set of all integers) with the property that $S_{f} \stackrel{\text { def }}{=}\{x \in X \mid f(x) \neq 0\}$ is finite is called an extended generalised multiset over $X$. The set of all extended generalised multisets over $X$ is denoted by $\mathbb{Z}_{\text {ext }}(X)$. Each function $f \in \mathbb{Z}_{\text {ext }}(X)$ is finitely supported with $\operatorname{supp}(f)=\operatorname{supp}\left(S_{f}\right)$. The set $\left(\mathbb{Z}_{\text {ext }}(X),+, \widetilde{\star}\right)$ is an invariant commutative group, where $f+g: X \rightarrow \mathbb{Z}$ is defined pointwise by $(f+g)(x)=f(x)+g(x)$ for all $x \in X$ and $\widetilde{\star}$ is the standard $S_{A}$-action on $\mathbb{Z}_{f_{S}}^{X}$.

Definition 13. Let $(G, \cdot \diamond)$ be an invariant group. A finitely supported subgroup of $G$ is a subgroup of $G$, which is also an element of $\wp_{f s}(G)$.

\section{Example 5.}

1. Let $(G, \cdot \diamond)$ be an invariant group. The centre of $G$ (namely, $C(G):=\{g \in G \mid g \cdot u=u \cdot g$ for all $u \in G\}$ ) is a finitely supported subgroup of $G$, and it is itself an invariant group because it is empty-supported as an element of $\wp(G)$.

2. Let $X$ be a finitely supported subset of $G$. The subgroup of $G$ generated by $X$ (denoted by $[X]$ ) is a finitely supported (by supp $(X)$ ) subgroup of $G$, but not itself an invariant group.

If $(G, \cdot, \diamond)$ is an invariant group, we denote by $L_{f_{s}}(G)$ the family of all finitely supported subgroups of $G$ ordered by inclusion.

\section{Theorem 17.}

- Let $(G, \cdot \diamond)$ be an invariant group. Then $\left(L_{f_{S}}(G), \star, \subseteq\right)$ is an invariant complete lattice, where $\subseteq$ represents the classical inclusion relation on $\wp(G)$ and $\star$ is the $S_{A}$-action on $\wp(G)$.

- $\quad$ Furthermore, if $\mathcal{F}=\left(H_{i}\right)_{i \in I}$ is a finitely supported family of finitely supported subgroups of $G$, then its least upper bound is $\left[\cup_{i \in I} H_{i}\right]$ which is supported by $\operatorname{supp}(\mathcal{F})$.

From Tarski's theorem (Theorem 12), we obtain the next result.

Corollary 6. Let $(G, \cdot \diamond)$ be an invariant group and $f: L_{f s}(G) \rightarrow L_{f s}(G)$ a finitely supported, order-preserving function. The set of all fixed points of $f$ is itself a finitely supported (by supp $(f)$ ) complete lattice.

Definition 14. Let $(G, \cdot \diamond)$ be an invariant group. A fuzzy set $\eta$ over the invariant set $G$ (i.e., a finitely supported function $\eta: G \rightarrow[0,1])$ is called a finitely supported fuzzy subgroup of $G$ if the following conditions are satisfied:

- $\quad \eta(x \cdot y) \geq \min \{\eta(x), \eta(y)\}$ for all $x, y \in G$;

- $\eta\left(x^{-1}\right) \geq \eta(x)$ for all $x \in G$.

Example 6. Let $(F(A), \mathrm{T}, \widetilde{\star})$ be the invariant free group over the set $A$ of atoms defined as in Example 4(2). For an element $[w]=\left[x_{1}^{\varepsilon_{1}} x_{2}^{\varepsilon_{2}} \ldots x_{k}^{\varepsilon_{k}}\right]$ in $F(A)$, we define $\operatorname{sum}([w])=\varepsilon_{1}+\varepsilon_{2}+$ $\ldots+\varepsilon_{k}$. Whenever $[w]=\left[w^{\prime}\right]$, we have $\operatorname{sum}([w])=\operatorname{sum}\left(\left[w^{\prime}\right]\right)$, and so sum is well defined. It can be proved that sum is an equivariant (empty-supported) group homomorphism between the invariant groups $F(A)$ and $\mathbb{Z}$ (the set of all integers being a trivial invariant group).

Let us consider $\eta_{A}: F(A) \rightarrow[0,1]$ defined by 


$$
\eta_{A}([w])= \begin{cases}0, & \text { if } \operatorname{sum}([w]) \text { is an odd integer; } \\ 1-\frac{1}{n}, & \text { if } \operatorname{sum}([w])=m \cdot 2^{n} \text { where } m \text { is an odd integer and } n \in \mathbb{N} \\ 1, & \text { ifsum }([w])=0 .\end{cases}
$$

It can be proved that $\mu_{A}$ is a finitely supported fuzzy subgroup of $F(A)$.

Theorem 18. Let $(G, \cdot, \diamond)$ be an invariant group. The set $F L_{f s}(G)$ consisting of all finitely supported fuzzy subgroups of $G$ forms an invariant complete lattice with respect to fuzzy sets inclusion.

In order to prove Theorem 18, the construction of least upper bounds for finitely supported subsets of $F L_{f_{s}}(G)$ follows the next steps [9]:

- $\quad$ First, we proved that $F L_{f_{s}}(G)$ is itself an invariant set; that is, we verified that $\pi \widetilde{\star} \mu$ is a finitely supported fuzzy subgroup of $G$ for all $\pi \in S_{A}$ and $\mu \in F L_{f_{s}}(G)$ (it satisfies the conditions in Definition 14$)$, where $\widetilde{\star}$ is the $S_{A}$-action on $[0,1]_{f_{S}}^{G}$.

- We remarked that the inclusion relation $\sqsubseteq$ on $F L_{f_{s}}(G)$, defined by $\mu \sqsubseteq \eta$ if and only if $\mu(x) \leq \eta(x)$ for all $x \in G$, is equivariant.

- $\quad$ For each $\alpha \in[0,1]$ and each $v \in[0,1]_{f s^{\prime}}^{G}$, we defined $G_{\alpha}^{v}=\{x \in G \mid v(x) \geq \alpha\}$ (which corresponds to the concept of $\alpha$-cut). We obtained that each $G_{\alpha}^{v}$ is finitely supported by $\operatorname{supp}(v)$.

- As in Example 5(2), we obtained that each subgroup $\left[G_{\alpha}^{v}\right]$ generated by $G_{\alpha}^{v}$ is finitely supported by $\sup p(v)$.

- For any finitely supported function $\mu: G \rightarrow[0,1]$, we defined the function $\mu^{*}: G \rightarrow$ $[0,1]$ by $\mu^{*}(x)=$ supremum $\left\{\alpha \in[0,1] \mid x \in\left[G_{\alpha}^{\mu}\right]\right\}$ for any $x \in G$, whereby supremum we denoted the least upper bounds in the set of real numbers. We proved that $\mu^{\star}$ is supported by $\operatorname{supp}(\mu)$.

- If $\mathcal{F}=\left(\mu_{i}\right)_{i \in I}$ is a finitely supported family of elements from $F L_{f s}(G)$, we defined $\bigsqcup_{i \in I} \mu_{i}: G \rightarrow[0,1]$ by $\underset{i \in I}{\sqcup} \mu_{i}(x)=\operatorname{supremum}\left\{\mu_{i}(x) \mid i \in I\right\}$ for all $x \in G$. Since $[0,1]$ is a $\mathrm{ZF}$ (trivial invariant) complete lattice, from Theorem 11 we have that $\operatorname{supp}(\mathcal{F})$ supports $\sqcup_{i \in I} \mu_{i}$. Therefore, we have that $\left(\sqcup_{i \in I} \mu_{i}\right)^{*}$ is finitely supported by $\operatorname{supp}\left(\sqcup_{i \in I} \mu_{i}\right) \subseteq$ $\operatorname{supp}(\mathcal{F})$.

- As in the standard fuzzy groups theory, we found that $\left(\sqcup_{i \in I} \mu_{i}\right)^{*}$ is a fuzzy subgroup of $G$ (in the sense of Definition 14) and it is the least upper bound of $\mathcal{F}$ in $F L_{f s}(G)$ with respect to the order relation $\sqsubseteq$.

From Tarski's theorem (Theorem 12), the next result follows.

Corollary 7. Let $(G, \cdot, \diamond)$ be an invariant group and $f: F L_{f_{s}}(G) \rightarrow F L_{f_{s}}(G)$ a finitely supported, order-preserving function. The set of all fixed points of $f$ is itself a finitely supported (by supp $(f)$ ) complete lattice.

Theorem 19. Let $(G, \cdot, \diamond)$ be an invariant group. The set $F N_{f s}(G)$ consisting of all finitely supported fuzzy normal subgroups of $G$ forms an invariant modular lattice with respect to fuzzy sets inclusion.

\section{T-Fuzzy Sets and Invariant Strong Inductive Sets}

We introduce the concept of the $T$-fuzzy set, where $T$ is an invariant partially ordered set having the property that every finitely supported totally ordered subset of $T$ has a least upper bound in $T$. We present some fixed point results in a more general framework; they can be also applied to T-fuzzy sets. 


\section{Definition 15.}

1. An invariant strong inductive set is an invariant partially ordered set $(T, \cdot, \sqsubseteq)$ with the property that every finitely supported totally ordered subset (i.e., every finitely supported chain) of $T$ has a least upper bound in $T$.

2. Let $\left(X, \cdot X, \sqsubseteq_{X}\right)$ and $\left(Y, \cdot Y, \sqsubseteq_{Y}\right)$ be two invariant partially ordered sets. A finitely supported function $f: X \rightarrow Y$ is c-continuous if and only if for each finitely supported, countable sequence $\left(u_{n}\right)_{n \in \mathbb{N}}$ in $X$ which has a least upper bound, we have that $f\left(\left(u_{n}\right)_{n \in \mathbb{N}}\right)$ has a least upper bound in $Y$ and $f\left(\underset{n \in \mathbb{N}}{\sqcup} u_{n}\right)=\underset{n \in \mathbb{N}}{\sqcup}\left(f\left(u_{n}\right)\right)$.

Definition 16. Let $(T, \cdot, \sqsubseteq)$ be an invariant strong inductive set and $(U, \diamond)$ an invariant set. A T-fuzzy set over $U$ is a finitely supported function $\mu: U \rightarrow T$.

The $T$-fuzzy sets are characterised by the following property.

\section{Theorem 20.}

- Let $(U, \diamond)$ be an invariant set and $(T, \cdot, \sqsubseteq)$ an invariant strong inductive set. The family of those finitely supported functions $f: U \rightarrow T$ (i.e., the family of all finitely supported $T$-fuzzy sets over $U$ ) is an invariant strong inductive set with the order relation $\leq$ defined by $f \leq g$ if and only if $f(x) \sqsubseteq g(x)$ for all $x \in U$.

- $\quad$ Furthermore, if $\mathcal{F}=\left(f_{i}\right)_{i \in I}$ is a finitely supported, totally ordered family of T-fuzzy sets over the invariant set $U$, its least upper bound with respect to $\leq$ is $\underset{i \in I}{\vee} f_{i}: U \rightarrow T$ defined by $\left(\underset{i \in I}{\vee} f_{i}\right)(x)=\sqcup_{i \in I}\left\{f_{i}(x) \mid i \in I\right\}$ for all $x \in U$, whereby $\sqcup$ we denoted least upper bounds in $T$ of finitely supported totally ordered subsets (with respect to $\sqsubseteq$ ).

The following theorem connects the concept of a 'uniformly supported set' with the concept of a 'invariant strong inductive set'.

\section{Theorem 21.}

- An invariant partially ordered set $(T, \cdot, \sqsubseteq)$ with the property that every uniformly supported subset of $T$ has a least upper bound in $T$ is an invariant strong inductive set.

- An invariant partially ordered lattice (not necessarily complete) $(T, \cdot, \sqsubseteq$ ) with the property that $T$ does not contain a uniformly supported, infinite subset is an invariant strong inductive set.

The following result presents a hierarchical construction of invariant sets containing no uniformly supported, infinite subsets [6,7]. We were able to prove this property for apparently large finitely supported sets that are presented as functions spaces.

\section{Theorem 22.}

1. Let $A \leq n=\left\{\left(a_{1}, \ldots, a_{k}\right) \mid a_{1}, \ldots, a_{k} \in A, k \leq n\right\}$. Let $T$ be a finitely supported subset of an invariant set such that $T$ does not contain a uniformly supported, infinite subset. The function space $T_{f s}^{A \leq n}$ does not contain a uniformly supported, infinite subset, whenever $n \in \mathbb{N}$.

2. Let $\wp_{\leq n}(A)=\left\{Z \in \wp_{\text {fin }}(A) \mid Z \in \wp_{m}(A)\right.$ for some $\left.m \leq n\right\}$, where $\wp_{m}(A)$ is the family of all $m$-sized subsets of $A$. Let $T$ be a finitely supported subset of an invariant set such that $T$ does not contain a uniformly supported, infinite subset. The function space $T_{f s}^{\wp \leq n}(A)$ does not contain a uniformly supported, infinite subset, whenever $n \in \mathbb{N}$.

Corollary 8. Let $T$ be a finitely supported subset of an invariant set such that $T$ does not contain a uniformly supported, infinite subset. For any $n \in \mathbb{N}$,

1. The function space $T_{f s}^{A^{n}}$ does not contain a uniformly supported, infinite subset;

2. The function space $T_{f s}^{\wp_{n}(A)}$ does not contain a uniformly supported, infinite subset. 
Corollary 9. Let $P$ be an invariant set (in particular, $P$ could be an invariant complete lattice or an invariant strong inductive set) that does not contain a uniformly supported, infinite subset. Let $X$ be one of the sets $A^{n}, A^{\leq n}, \wp_{n}(A), \wp_{\leq n}(A)$ for some $n \in \mathbb{N}$. The set of all $P$-fuzzy sets over the invariant set $X$ does not contain a uniformly supported, infinite subset.

The following four results are specific to finitely supported sets, i.e., they do not have ZF correspondents. We present some examples of finite powersets that are invariant strong inductive sets. Such a result does not hold in ZF since a ZF set could admit an unbounded countable ascending chain of finite subsets.

Theorem 23. Let $(T, \cdot)$ be an invariant set such that $T$ does not contain a uniformly supported, infinite subset. Then $\left(\wp_{\text {fin }}(T), \star, \subseteq\right)$ does not contain a uniformly supported, infinite subset, and so it is an invariant strong inductive set.

Corollary 10. Let $(T, \cdot)$ be an invariant set such that $T$ does not contain a uniformly supported, infinite subset. Then $\left(\wp_{f i n}\left(T_{f_{S}}^{A^{n}}\right), \star, \subseteq\right)$ is an invariant strong inductive set, $n \in \mathbb{N}$.

Corollary 11. Let $(T, \cdot)$ be an invariant set such that $T$ does not contain a uniformly supported, infinite subset. Then $\left(\wp_{f i n}\left(T_{f_{S}}^{\wp_{n}(A)}\right), \star, \subseteq\right)$ is an invariant strong inductive set, $n \in \mathbb{N}$.

Corollary 12. Let $(T, \cdot)$ be an invariant set such that $T$ does not contain a uniformly supported, infinite subset. Then $\left(\wp_{f i n}\left(\wp_{f s}\left(A^{n}\right)\right), \star, \subseteq\right)$ is an invariant strong inductive set, $n \in \mathbb{N}$.

\section{Example 7.}

- Let $X$ be an invariant set. The function $f: X \rightarrow \wp_{\text {fin }}(A)$ defined by $f(x)=\operatorname{supp}(x)$ for all $x \in X$ is an equivariant T-fuzzy set over $X$. This is because $\left(\wp_{\text {fin }}(A), \star, \subseteq\right)$ is an invariant strong inductive set according to Theorem 23 and, for all $\pi \in S_{A}$, we have $\operatorname{supp}(\pi \cdot x)=\pi(\operatorname{supp}(x))=\pi \star \operatorname{supp}(x)$.

- Let $(X, \cdot)$ be an invariant set which does not contain an infinite uniformly supported subset. According to Theorem $23,\left(\wp_{\text {fin }}(X), \star, \subseteq\right)$ is an invariant, strong inductive set. Let $F A S(X)=\{f: X \rightarrow[0,1] \mid F Z S(X, f)$ is finite $\}$. Since $[0,1]$ is a trivial invariant complete lattice, according to Theorem 10(2), every function $f \in F A S(X)$ is finitely supported. As in Example 3(2), for all $\pi \in S_{A}$, we have $F Z S(X, \pi \widetilde{\star} f)=\pi \star F Z S(X, f)$ for all $f \in F A S(X)$, and so $F A S(X)$ is an invariant set. The equivariant function $\psi$ between the invariant set FAS $(X)$ and the invariant set $\left(\wp_{\text {fin }}(X), \star\right)$ defined by $\psi(f)=F Z S(X, f)$ for all $f \in F A S(X)$ is a $T$-fuzzy set over FAS $(X)$.

- Let $(X, \cdot)$ be an invariant set which does not contain an infinite uniformly supported subset. On FAS $(X)$ we define the relation $\sim b y: f \sim g$ if and only if $F Z S(X, f)=F Z S(X, g)$. Then, according to Corollary 3 , since equivariant isomorphisms of monoids are also equivariant functions, we know that there is an equivariant function $\varphi$ between the invariant set $F A S(X) / \sim$ and the invariant set $\left(\wp_{\text {fin }}(X), \star\right)$ defined by $\varphi([f])=F Z S(X, f)$ for all $f \in F A S(X)$, where by $[f]$ we denoted the family of functions from $X$ to $[0,1]$ having the same algebraic support as $f$. Thus, $\varphi$ is a T-fuzzy set over FAS $(X) / \sim$.

From Theorem 20, Theorem 23, Corollary 10, Corollary 11 and Theorem 22, the following property of $T$-fuzzy sets can be presented.

Theorem 24. Let $(U, \diamond)$ be an invariant set.

1. Let $T$ be an invariant set such that $T$ does not contain a uniformly supported, infinite subset. The family of all $\wp_{\text {fin }}(T)$-fuzzy sets over the invariant set $U$ is an invariant strong inductive set with the order relation $\leq$ defined by $f \leq g$ if and only if $f(x) \subseteq g(x)$ for all $x \in U$.

2. Let $(T, \cdot)$ be an invariant set such that $T$ does not contain a uniformly supported, infinite subset. For each $n \in \mathbb{N}$, the family of all $\wp_{f i n}\left(T_{f_{s}}^{A^{n}}\right)$-fuzzy sets over the invariant set $U$ is 
an invariant strong inductive set with the order relation $\leq$ defined by $f \leq g$ if and only if $f(x) \subseteq g(x)$ for all $x \in U$.

3. Let $(T, \cdot)$ be an invariant set such that $T$ does not contain a uniformly supported, infinite subset. For each $n \in \mathbb{N}$, the family of all $\wp_{\text {fin }}\left(T_{f s}^{\wp_{n}}(A)\right)$-fuzzy sets over the invariant set $U$ is an invariant strong inductive set with the order relation $\leq$ defined by $f \leq g$ if and only if $f(x) \subseteq g(x)$ for all $x \in U$.

According to Theorem 20, the following fixed point results can provide properties of finitely supported $T$-fuzzy sets over an invariant set. We chose to present the results in the general case, making them applicable also for other finitely supported structures.

The Bourbaki-Witt theorem is an important fixed-point result in mathematics. Its ZF formulation is used to define recursive data types (e.g., linked lists in domain theory). Other applications can be found in logic or in the theory of computable functions. This theorem is also valid for finitely supported progressive self-functions on invariant sets.

Theorem 25 (Bourbaki-Witt Theorem for fss). Let ( $T, \cdot, \sqsubseteq)$ be an invariant strong inductive set. Let $f: T \rightarrow T$ be a finitely supported function having the additional property that $x \sqsubseteq f(x)$ for all $x \in T$. Then there is $u \in T$ such that $f(u)=u$.

In terms of $T$-fuzzy sets, this result states that, if $(T, \cdot, \sqsubseteq)$ is an invariant strong inductive set and $f$ is a $T$-fuzzy set over the invariant set $T$ with the additional property that $x \sqsubseteq f(x)$ for all $x \in T$, then $f$ has a fixed point.

Corollary 13. Let $(T, \cdot, \sqsubseteq)$ be an invariant strong inductive set. Let $f: T \rightarrow T$ be a finitely supported function having the additional property that $x \sqsubseteq f(x)$ for all $x \in T$. Then for any $y \in T$, there is $u \in T$ such that $f(u)=u$ and $y \sqsubseteq u$.

If in the statement of Theorem 25 we impose the requirement regarding the existence of least upper bounds for all uniformly supported subsets of an invariant set (instead of for all finitely supported totally ordered subsets of an invariant set), we obtain the following result of Bourbaki-Witt type [6,10].

Theorem 26. Let $(T, \cdot, \sqsubseteq)$ be a non-empty invariant partially ordered set with the property that every uniformly supported subset of $T$ has a least upper bound. Let $f: T \rightarrow T$ be a finitely supported function having the additional property that $x \sqsubseteq f(x)$ for all $x \in T$. Then there is $u \in T$ such that $f(u)=u$.

We proved in [6] that the existence of fixed points for a finitely supported, orderpreserving function is possible even in the case when the related function is defined on an invariant strong inductive set (instead on an invariant complete lattice).

Theorem 27 (Tarski-Extended Theorem for fss). Let ( $T, \cdot, \sqsubseteq$ ) be an invariant strong inductive set. Let $f: T \rightarrow T$ be a finitely supported, order preserving function having the additional property that there is $x_{0} \in T$ such that $x_{0} \sqsubseteq f\left(x_{0}\right)$. Then there is $u \in T$ such that $f(u)=u$.

In terms of $T$-fuzzy sets, this result states that, if $(T, \cdot, \sqsubseteq)$ is an invariant strong inductive set and $f$ is an order-preserving $T$-fuzzy set over the invariant set $T$ having the additional property that $x_{0} \in T$ with $x_{0} \sqsubseteq f\left(x_{0}\right)$ exists, then $f$ has a fixed point.

From Theorems 20, 25 and 27 we obtain the following fixed point result for $T$-fuzzy sets.

Theorem 28. Let $(U, \diamond)$ be an invariant set and $(T, \cdot, \sqsubseteq)$ an invariant strong inductive set.

1. Let $\varphi: T_{f_{s}}^{U} \rightarrow T_{f_{s}}^{U}$ be a finitely supported function with the property that $f \leq \varphi(f)$ for all $f \in T_{f_{s}}^{U}$, where $\leq$ is the order relation on the family of all $T$-fuzzy sets over the invariant set 
$U$ defined by $f_{1} \leq f_{2}$ if and only if $f_{1}(x) \sqsubseteq f_{2}(x)$ for all $x \in U$. Then there is $g \in T_{f s}^{U}$ such that $\varphi(g)=g$.

2. Let $\varphi: T_{f s}^{U} \rightarrow T_{f_{s}}^{U}$ be a finitely supported, order-preserving function with the property that $f_{0} \in T_{f_{s}}^{U}$ exists such that $f_{0} \leq \varphi\left(f_{0}\right)$, where $\leq$ is the order relation on the family of all $T$-fuzzy sets over the invariant set $U$ defined by $f_{1} \leq f_{2}$ if and only if $f_{1}(x) \sqsubseteq f_{2}(x)$ for all $x \in U$. Then $g \in T_{f_{s}}^{U}$ is with $f_{0} \sqsubseteq g$ such that $\varphi(g)=g$.

In ZF, the following two fixed point theorems (known as the Tarski-Kantorovitch theorem and Scott theorem, respectively) have applications in domain theory, in formal semantics of programming languages, in the theory of iterated function systems and in abstract interpretation. We adequately reformulate them for finitely supported sets.

Theorem 29 (Tarski-Kantorovitch Theorem for fss). Let ( $T, \cdot, \sqsubseteq$ ) be an invariant partially ordered set and $f: T \rightarrow T$ a finitely supported c-continuous function. Assume that $x_{0} \in T$, having the following properties:

- $\quad x_{0} \sqsubseteq f\left(x_{0}\right)$;

- Every finitely supported countable chain in $\uparrow_{x_{0}}=\left\{x \in T \mid x_{0} \sqsubseteq x\right\}$ has a least upper bound in $T$.

Then $f$ has a fixed point $u=\underset{n \in \mathbb{N}}{\sqcup} f^{n}\left(x_{0}\right)$ with the property that $\operatorname{supp}(u) \subseteq \operatorname{supp}(f) \cup$ $\operatorname{supp}\left(x_{0}\right)$.

Corollary 14 (Scott Theorem for fss). Let $(T, \cdot, 5,0)$ be an invariant, partially ordered set with a least element 0 and with the additional property that any finitely supported countable ascending chain in $T$ has a least upper bound. Every finitely supported c-continuous function $f: T \rightarrow T$ has a least fixed point $u=\underset{n \in \mathbb{N}}{\sqcup} f^{n}(0)$ with the property that $\operatorname{supp}(u) \subseteq \operatorname{supp}(f)$.

Corollary 15. Let $(T, \cdot, \sqsubseteq, 0)$ be an invariant strong inductive set with a least element 0 . Every finitely supported c-continuous function $f: T \rightarrow T$ has a least fixed point $u=\underset{n \in \mathbb{N}}{\sqcup} f^{n}(0)$ with the property that $\operatorname{supp}(u) \subseteq \operatorname{supp}(f)$.

From Corollary 15 we conclude that if $(T, \cdot, \sqsubseteq, 0)$ is an invariant strong inductive set with a least element 0 and $f$ is an $c$-continuous $T$-fuzzy set over the invariant set $T$, then $\sqcup{ }_{n \in \mathbb{N}} f^{n}(0)$ is the least fixed point of $f$.

Theorem 29 was generalised in [10] to the following result.

Theorem 30. Let $(T, \cdot, \sqsubseteq)$ be an invariant partially ordered set with the property that every uniformly supported subset has a least upper bound. If $f: T \rightarrow T$ is a finitely supported ccontinuous function having the additional property that $x_{0} \in T$ and $k \in \mathbb{N}^{*}$ such that $x_{0} \sqsubseteq f^{k}\left(x_{0}\right)$, then $\underset{n \in \mathbb{N}}{\sqcup} f^{n}\left(x_{0}\right)$ is a fixed point of $f$.

Proposition 7. Let $(T, \cdot, \sqsubseteq)$ be an invariant partially ordered set containing no uniformly supported, infinite subset and $f: T \rightarrow T$ a finitely supported, order-preserving function over $T$.

- If the set $X=\{x \in T \mid x \sqsubseteq f(x)\}$ is non-empty and totally ordered, then $f$ has the greatest fixed point defined as $\operatorname{gfp}(f)=\sqcup X$.

- If the set $X^{\prime}=\{x \in T \mid f(x) \sqsubseteq x\}$ is non-empty and totally ordered, then $f$ has the least fixed point defined as $\operatorname{lfp}(f)=\sqcap X^{\prime}$.

In either of the above cases, $f$ only has many finitely fixed points that form a finitely supported complete lattice.

We presented above examples of invariant partially ordered sets that do not contain uniformly supported, infinite subsets. For these sets, some fixed point properties hold. 
Theorem 31. Let $(T, \cdot, \sqsubseteq)$ be an invariant partially ordered set that does not contain a uniformly supported, infinite subset. Let $f: T \rightarrow T$ be a finitely supported function having the additional property that $x \sqsubseteq f(x)$ for all $x \in T$. Then for each $x \in T n \in \mathbb{N}$ exists, such that $f^{k}(x)$ is a fixed point of $f$ for all $k \geq n$.

Theorem 32. Let $(T, \cdot, \sqsubseteq)$ be an invariant, partially ordered set that does not contain a uniformly supported, infinite subset. Let $f: T \rightarrow T$ be a finitely supported, order-preserving function having the additional property that there is $x_{0} \in T$ such that $x_{0} \sqsubseteq f\left(x_{0}\right)$. Then there is $n \in \mathbb{N}$ such that $f^{k}\left(x_{0}\right)$ is a fixed point of $f$ for all $k \geq n$.

From Theorems 22, 23, 31 and 32, we obtain the following corollaries.

Corollary 16. Let $(T, \cdot)$ be an invariant set such that $T$ does not contain a uniformly supported, infinite subset. Let $f: \wp_{\text {fin }}(T) \rightarrow \wp_{\text {fin }}(T)$ be a $\wp_{\text {fin }}(T)$-fuzzy set over the invariant set $\wp_{\text {fin }}(T)$ and let $T_{0} \in \wp_{\text {fin }}(T)$ such that $T_{0} \subseteq f\left(T_{0}\right)$. If $f$ is order-preserving or progressive (i.e., $f$ has the property that $Y \subseteq f(Y)$ for all $Y \in \wp_{\text {fin }}(T)$ ), then $n \in \mathbb{N}$ exists, such that $f^{k}\left(T_{0}\right)$ is a fixed point of $f$ for all $k \geq n$.

Corollary 17. Let $(T, \cdot)$ be an invariant set such that $T$ does not contain a uniformly supported, infinite subset. Let $f$ be a $\wp_{f i n}\left(T_{f s}^{A^{n}}\right)$-fuzzy set over the invariant set $\wp_{f i n}\left(T_{f s}^{A^{n}}\right)$ having the additional property that $f$ is a progressive function. Then $T_{0} \in \wp_{\text {fin }}\left(T_{f_{s}}^{A^{n}}\right)$ such that $f\left(T_{0}\right)=T_{0}$.

Corollary 18. Let $(T, \cdot)$ be an invariant set such that $T$ does not contain a uniformly supported, infinite subset. Let $f$ be a $\wp_{\text {fin }}\left(T_{f_{s}}^{A^{n}}\right)$-fuzzy set over the invariant set $\wp_{\text {fin }}\left(T_{f_{s}}^{A^{n}}\right)$ which is orderpreserving. Then a least $T_{0} \in \wp_{\text {fin }}\left(T_{f_{s}}^{A^{n}}\right)$ supported by supp $(f)$ such that $f\left(T_{0}\right)=T_{0}$.

Corollary 19. Let $(T, \cdot)$ be an invariant set such that $T$ does not contain a uniformly supported, infinite subset. Let $f$ be a $\wp_{\text {fin }}\left(T_{f_{s}}^{\wp_{n}(A)}\right)$-fuzzy set over the invariant set $\wp_{f i n}\left(T_{f_{s}}^{\wp_{n}(A)}\right)$ having the additional property that $f$ is a progressive function. Then $T_{0} \in \wp_{f i n}\left(T_{f_{S}}^{\wp_{n}(A)}\right)$ such that $f\left(T_{0}\right)=T_{0}$.

Corollary 20. Let $(T, \cdot)$ be an invariant set such that $T$ does not contain a uniformly supported, infinite subset. Let $f$ be a $\wp_{f i n}\left(T_{f_{s}}^{\wp_{n}(A)}\right)$-fuzzy set over the invariant set $\wp_{f i n}\left(T_{f_{s}}^{\wp_{n}(A)}\right)$ which is order-preserving. Then there is a least $T_{0} \in \wp_{f i n}\left(T_{f_{S}}^{\wp_{n}(A)}\right)$ supported by supp $(f)$ such that $f\left(T_{0}\right)=T_{0}$.

For a particular class of $T$-fuzzy sets, i.e., for those $\wp_{\text {fin }}(A)$-fuzzy sets over the invariant set $\wp_{\text {fin }}(A)$ (which are actually finitely supported self-functions defined on the finite powerset of atoms) that satisfy some additional conditions such as injectivity, surjectivity, monotony or progressivity, we were able to prove stronger fixed point properties than in the general case; we mention some of them here.

Proposition 8. Let $f$ be a $\wp_{\text {fin }}(A)$-fuzzy set over the invariant set $\wp_{\text {fin }}(A)$ which is strictly order-preserving (i.e., $f$ has the property that $U \subsetneq V$ implies $f(U) \subsetneq f(V)$ ). Then we have $Z \backslash \operatorname{supp}(f)=f(Z \backslash \operatorname{supp}(f))$ for all $Z \in \wp_{\text {fin }}(A)$.

Proposition 9. Let $f$ be a $\wp_{\text {fin }}(A)$-fuzzy set over the invariant set $\wp_{\text {fin }}(A)$ with the property that $Y \subseteq f(Y)$ for all $Y \in \wp_{\text {fin }}(A)$. There are infinitefixed points of $f$, namely, those finite subsets of $A$ containing all the elements of $\operatorname{supp}(f)$. 
Proposition 10. Let $f$ be a $\wp_{\text {fin }}(A)$-fuzzy set over the invariant set $\wp_{\text {fin }}(A)$ which is injective. For each $Y \in \wp_{\text {fin }}(A)$ we have $Y \backslash \sup p(f) \neq \varnothing$ if and only if $f(Y) \backslash \sup p(f) \neq \varnothing$. Furthermore, $Y \backslash \operatorname{supp}(f)=f(Y) \backslash \operatorname{supp}(f)$.

Proposition 11. Let $f$ be a $\wp_{\text {fin }}(A)$-fuzzy set over the invariant set $\wp_{\text {fin }}(A)$ which is surjective. For each $Y \in \wp_{\text {fin }}(A)$ we have $Y \backslash \sup p(f) \neq \varnothing$ if and only if $f(Y) \backslash \sup p(f) \neq \varnothing$. Furthermore, $Y \backslash \operatorname{supp}(f)=f(Y) \backslash \operatorname{supp}(f)$.

Proposition 12. Let $f$ be a $\wp_{\text {fin }}(A)$-fuzzy set over the invariant set $\wp_{\text {fin }}(A)$ having the properties that $f(Y) \subseteq Y$ for all $Y \in \wp_{\text {fin }}(A)$ and $f(Y) \neq \varnothing$ for all $Y \neq \varnothing$. Then $f(Z)=Z$ for all $Z \in \wp_{\text {fin }}(A)$ with $Z \cap \operatorname{supp}(f)=\varnothing$.

From Theorems 20 and 14 we obtain the following fixed point result for $T$-fuzzy sets.

Theorem 33. Let $(U, \diamond)$ be an invariant set and $(T, \cdot, \sqsubseteq)$ an invariant, strong inductive set with a least element 0 . Let $\varphi: T_{f s}^{U} \rightarrow T_{f s}^{U}$ be a finitely supported, c-continuous function defined on the family of all $T$-fuzzy sets over the invariant set $U$. Then a least $g \in T_{f_{s}}^{U}$ with the property that $\varphi(g)=g$. Furthermore, $g=\vee_{n \in \mathbb{N}} \varphi^{n}\left(0_{U}\right)$, whereby $\vee$ we denoted the least upper bounds in $T_{f s}^{U}$ with respect to the relation $\leq$ defined by $f_{1} \leq f_{2}$ if and only if $f_{1}(x) \sqsubseteq f_{2}(x)$ for all $x \in U$, and $0_{U}: U \rightarrow T, 0_{U}(x)=0$ for all $x \in U$.

\section{Conclusions}

This article represents an overview of the properties of $L$-fuzzy sets and $T$-fuzzy sets over possibly infinite universes, properties presented in a discrete manner by involving the notion of finite support. We presented a relationship between the algebraic support and the finite support of an (L-)fuzzy set over an invariant set. We translated several concepts from the framework of classical ZF fuzzy sets (such as $\alpha-c u t$, operations with fuzzy sets, fuzzy extension principles, fuzzy subgroups) into the framework of finitely supported structures, and proved the consistency of their related results in the new framework of finitely supported structures.

Several fixed-point theorems for partially ordered sets (that can be particularly applied to the families of $L$-fuzzy sets and $T$-fuzzy sets over invariant sets) are adequately reformulated in the framework of finitely supported sets; they can also be generalised by imposing requirements only for uniformly supported subsets. Also presented are other fixed point properties for functions defined on invariant sets containing no uniformly supported, infinite subsets. Specific properties of self-functions defined on finite powersets are presented as corollaries of some general results. We presented even stronger fixed-point properties for order preserving, injective, surjective or progressive self-functions defined on the finite powerset of atoms. We introduced and described lattices and inductive sets in the framework of finitely supported structures. We connected the concept of $L$-fuzzy set with the concept of invariant complete lattice, and the concept of T-fuzzy set with the concept of invariant strong inductive set. Some particular invariant complete lattices were studied. We mentioned the finitely supported subsets of an invariant set, the finitely supported functions from an invariant set to an invariant complete lattice (i.e., the finitely supported $L$-fuzzy sets with $L$ being an invariant complete lattice) and the finitely supported (fuzzy) subgroups of an invariant group. For these particular invariant complete lattices, the theorems presented in this article can provide new properties. We also presented some examples of invariant strong inductive sets, such as the finite powerset of a set containing no uniformly supported, infinite subset. For the finitely supported selffunctions on invariant strong inductive sets, some fixed point properties are mentioned. The related fixed-point properties (presented in the general case) lead to applications in the theory of $L$-fuzzy sets and T-fuzzy sets over invariant sets (e.g., Theorems 15, 28 and 33). According to Examples 3 and 7, the functions which associate with any classical fuzzy set on an invariant set means its algebraic support or its finite support are fss $L$-fuzzy 
sets, and in some cases, fss T-fuzzy sets. In this way, we can discretely model the infinite classical fuzzy sets over invariant sets in terms of finite supports or algebraic supports using the properties of fss $L$-fuzzy sets or fss $T$-fuzzy sets, respectively.

\section{Future Research}

We mention below some future work directions.

- Finitely supported monoids can be used to describe automata and languages over infinite alphabets. A relaxed notion of 'finite' called 'orbit finite' is defined; it means 'having a finite number of orbits (equivalence classes) under a certain group action' [15]. A future goal is to describe and study finitely supported $M$-fuzzy sets, where $M$ is a finitely supported monoid (similarly to finitely supported $T$-fuzzy sets) and finitely supported fuzzy submonoids (similarly to finitely supported fuzzy sugbroups). For these fuzzy structures, we would provide embedding theorems, isomorphism properties, universality theorems and applications in automata theory and programming languages.

- The study of fixed points is important since they can encode recursion or model inductive reasoning. Other applications can be found in the theory of computable functions, in logic, in abstract interpretation to prove the existence of least fixed points for specific mappings (defined on chain complete sets of properties) modelling the transitions between properties of programming languages, in formal semantics of programming languages and in the theory of iterated function systems. A fixed-point induction technique in the framework of finitely supported structures could be presented, to prove even stronger properties than those that would lead to usual replacement of 'non-atomic structure' with 'atomic, finitely supported structure' in a related ZF result. For example, a fixed-point theorem of Knaster-Tarski type claims that a finitely supported, monotone self-function defined on a finitely supported partially ordered set having the property that any finitely supported subset has a least upper bound is valid in fss if we require the existence of least upper bounds only for uniformly supported subsets, and not for all finitely supported subsets of the domain of the related function.

- We intend to present some examples of apparently large sets (such as finite powersets or functions spaces) that satisfy some Dedekind-finiteness properties and for which the fss fixed-point properties can provide a certain form of stability. We particularly mention $\wp_{f i n}(X), \wp_{f i n}\left(X_{f s}^{A^{\leq n}}\right), \wp_{f i n}\left(X_{f s}^{\wp \leq n}(A)\right)$ that are proved to be fss Dedekind finite whenever $X$ is a finitely supported Dedekind finite set (i.e., whenever $X$ has the property that every finitely supported injection $f: X \rightarrow X$ is also surjective). Many other pair-wise, non-equivalent forms of infinity such as Levy infinity, Tarski infinity, Kuratowski infinity, Mostowski infinity, ascending infinity, etc. can be defined and compared; for functions on sets satisfying these forms of infinity, new calculability and stability properties could be presented.

- Uncertainty is an inherent property of all living systems. P systems are models used in membrane computing inspired by the behaviour of living cells [16]. There have been a few defined fuzzy P systems: fuzzy cell-like P systems and fuzzy reasoning spiking neural $P$ systems. Fuzzy aspects have been used to handle the uncertainty in the number of copies of the reactants, imperfectness of objects in membranes and approximate copies of reactants used in reactions. A possible future work is to continue the existing development by introducing fss $L$-fuzzy sets in membrane computing, and studying them together with the natural finiteness properties.

Author Contributions: All authors contributed equally to this work. All authors have read and agreed to the published version of the manuscript.

Funding: This research received no external funding.

Conflicts of Interest: The authors declare no conflict of interest. 


\section{References}

1. Zadeh, L.A. Fuzzy sets. Inf. Control 1965, 8, 338-353. [CrossRef]

2. Zimmermann, H.J. Fuzzy Set Theory and Its Applications; Springer: Dordrecht, The Netherlands, 2001.

3. Barwise, J. Admissible Sets and Structures: An Approach to Definability Theory; Perspectives in Math. Logic; Springer: Berlin/Heidelberg, Germany, 1975; Volume 7.

4. Pitts, A.M. Nominal Sets Names and Symmetry in Computer Science; Cambridge University Press: Cambridge, UK, 2013.

5. Alexandru, A.; Ciobanu, G. Finitely Supported Mathematics: An Introduction; Springer: Cham, Switzerland, 2016.

6. Alexandru, A.; Ciobanu, G. Foundations of Finitely Supported Structures: A Set Theoretical Viewpoint; Springer: Cham, Switzerland, 2020.

7. Alexandru, A.; Ciobanu, G. Properties of the atoms in finitely supported structures. Arch. Math. Logic 2020, 59, 229-256. [CrossRef]

8. Alexandru, A.; Ciobanu, G. Fuzzy sets within finitely supported mathematics. Fuzzy Sets Syst. 2018, 339, 119-133. [CrossRef]

9. Alexandru, A.; Ciobanu, G. Fixed point results for finitely supported algebraic structures. Fuzzy Sets Syst. 2020, 397, 1-27. [CrossRef]

10. Alexandru, A.; Ciobanu, G. Uniformly supported sets and fixed points properties. Carpath. J. Math. 2020, 36, 351-364. [CrossRef]

11. Alexandru, A.; Ciobanu, G. Abstract interpretations in the framework of invariant sets. Fundam. Inf. 2016, 144, 1-22. [CrossRef]

12. Alexandru, A.; Ciobanu, G. Mathematics of multisets in the Fraenkel-Mostowski framework. Bull. Math. Soc. Sci. Math. Roumanie 2015, 58, 3-18.

13. Rosenfeld, A. Fuzzy groups. J. Math. Anal. Appl. 1971, 35, 512-517. [CrossRef]

14. Mordeson, J.N.; Bhutan, K.R.; Rosenfeld, A. Fuzzy Group Theory; Studies in Fuzziness and Soft Computing 182; Springer: Berlin/Heidelberg, Germany, 2005.

15. Bojanczyk, M.; Klin, B.; Lasota, S. Automata with group actions. In Proceedings of the 26th Symposium on Logic in Computer Science, Toronto, ON, Canada, 21-24 June 2011; pp. 355-364.

16. Păun, G.; Rozenberg, G.; Salomaa, A. (Eds.) Handbook of Membrane Computing; Oxford University Press: Oxford, UK, 2010. 